Experimenten hergeleiteten Werten, für „Mt. Ayliff“.

$$
t=2,9 \cdot 10^{16} \mathrm{sec}=9,2 \cdot 10^{8} \text { Jahre. }
$$

Dieser Zeitraum muß nicht notwendigerweise mit dem Alter, wie man es z. B. mit der Kalium-ArgonMethode erhält ${ }^{20}$, identisch sein, denn es ist durchaus denkbar, daß der für den Ursprung der Meteorite verantwortliche Himmelskörper erst längere Zeit

50 H. H. Uhlig, Geochim. Cosmochim. Acta 6, 282 [1954].

51 H. H. Uhlig, Geochim. Cosmochim. Acta 7, 34 [1955]. nach dem Erkalten in kleinere Bruchstücke zerfallen ist, ohne daß sich diese dabei bis zu ihrem Schmelzpunkt erwärmt hatten. Eine solche Entstehung der Meteorite ist nach metallurgischen Studien ${ }^{50,51}$ sogar ziemlich wahrscheinlich, auch ist die Bildung der Widmannstätten-Figuren an so kleinen Körpern, wie es die Meteorite sind, sonst nicht erklärbar.

Herrn Professor F. A. Paneth danken wir aufrichtig für die weitgehende Förderung, die er uns im Laufe dieser Arbeit zuteil werden ließ.

\title{
Massenspektrometer mit Doppelfokussierung zweiter Ordnung
}

\author{
Von H. Hintenberger und L. A. König \\ Aus dem Max-Planck-Institut für Chemie, Mainz \\ (Z. Naturforschg. 12 a, 773-785 [1957] ; eingegangen am 22. Juli 1957)
}

Herrn Professor F. A. Paneth zum 70. Geburtstag

\begin{abstract}
Es werden Massenspektrometer mit vollständiger Doppelfokussierung zweiter Ordnung, d. h. mit korrigiertem Öffnungsfehler $\left(\alpha^{2}\right)$, korrigierter gemischt öffnungswinkel- und geschwindigkeitsabhängiger Aberration $(\alpha \beta)$ und mit korrigierter rein geschwindigkeitsabhängiger Aberration $\left(\beta^{2}\right)$ angegeben. Ihre Abmessungen stellen die Lösungen eines komplizierten Gleichungssystems von 5 Gleichungen dar. 3 von den 8 in die Gleichungen eingehenden Unbekannten (Ablenkwinkel $\varphi_{\mathrm{m}}$ und Einund Austrittswinkel $\varepsilon^{\prime}$ und $\varepsilon^{\prime \prime}$ des Hauptstrahls am Magnetfeld) werden vorgegeben und die Abhängigkeit der 5 anderen Größen von diesen 3 Winkeln untersucht. Die Bewältigung der dabei auftretenden sehr umfangreichen numerischen Rechnungen wurde durch die Anwendung eines IBM-Magnettrommelrechners Type 650 ermöglicht. Das benutzte Rechenverfahren wird skizziert.

Es zeigt sich, daß sowohl für gleichsinnige als auch für gegensinnige Ablenkung im elektrischen und magnetischen Feld Massenspektrometer mit vollständiger Doppelfokussierung zweiter Ordnung möglich sind, über die an Hand von Tabellen und Diagrammen ein Úberblick gegeben wird. Besonders bei gleichsinniger Ablenkung in beiden Feldern ergeben sich Apparate mit vernünftigen Abmessungen. Vier solche Beispiele waren bereits in einer früheren Notiz (Z. Naturforschg. 12 a, 443 [1957]) veröffentlicht worden.
\end{abstract}

Mit der fortschreitenden Entwicklung massenspektroskopischer Methoden und der Verbreitung ihrer verschiedenen Anwendungen steigen ständig die Ansprüche an diese Instrumente sowohl hinsichtlich der Lichtstärke als auch in bezug auf das Auflösungsvermögen. Im Zuge dieser Entwicklung hat sich das Prinzip der Doppelfokussierung, das 1929 in einem Spezialfall von Bartky und Dempster ${ }^{1}$ und dann nach der Entwicklung einer allgemeinen ionenoptischen Theorie der Doppelfokussierung erter Ordnung durch Mattauch und Herzog ${ }^{2}$ von liesen und auch von anderen Forschern ${ }^{3,4,5,6}$ prakisch angewandt worden ist, durchgesetzt. Es handelt

\footnotetext{
1 W. Bartky u. A. J. Dempster, Phys. Rev. 33, 1019 [1929].

- J. Mattauch u. R. Herzog, Z. Phys. 89, 786 [1934].

3 A. J. Dempster, Proc. Amer. Phil. Soc. 75, 755 [1935].

${ }^{4}$ K. T. Bainbridge u. E. B. Jordan, Phys. Rev. 50, 282 [1936].
}

sich dabei aber stets um Instrumente, bei denen Doppelfokussierung nur in erster Näherung erreicht wird. In diesen Instrumenten treten Bildfehler auf, von denen diejenigen, die vom Öffnungswinkel $\alpha$ des Strahlenbündels und der relativen Geschwindigkeitsabweichung $\beta=\Delta v / v_{0}$ der Ionen von einem Normalwert $v_{0}$ quadratisch abhängen, am stärksten ins Gewicht fallen. Die Korrektur dieser Bildfehler stellt eine weitere wesentliche Verbesserungsmöglichkeit für Massenspektrographen und Massenspektrometer dar. In der vorliegenden Arbeit sollen die Möglichkeiten für die Konstruktion solcher Instrumente mit korrigierten Bildfehlern, die also voll-

5 a) C. Reuterswärd, Ark. Mat. Astr. Fys., K. Svenska Wet. Akad. 30 A, No. 7 [1943] ; b) H. Ewald, Z. Naturforschg. 1, 131 [1946]; c) N. B. Hannay, Rev. Sci. Instrum. 25, 644 [1954].

6 Zusammenfassend. H. Ewald u. H. Hintenberger, „Methoden und Anwendungen der Massenspektroskopie“", Verlag Chemie, Weinheim 1953. 
ständige Doppelfokussierung zweiter Ordnung aufweisen, näher untersucht werden. Dabei beschränken wir uns auf die Berechnung der Bahnen in der Mittelebene der Felder.

\section{Frühere Arbeiten}

Nachdem der Öffnungsfehler im elektrischen Radialfeld für einen Ablenkwinkel $\varphi_{\mathrm{e}}=\pi / \sqrt{2}$ bereits von Hughes und Rojansky ${ }^{7}$ angegeben wurde, sind die ionenoptischen Bildfehler für beliebige Ablenkwinkel in solchen Feldern von Johnson und NieR ${ }^{8}$ und von Voorhies ${ }^{9}$ berechnet worden. Auch in einer Arbeit von Marschall ${ }^{10}$ über die ionenoptischen Abbildungseigenschaften überlagerter elektrischer und magnetischer Felder sind Ausdrücke für die Bildfehler des Zylinderkondensators als Spezialfälle enthalten.

Für das geradlinig begrenzte homogene magnetische Sektorfeld ist der Öffnungsfehler bei senkrechtem Ein- und Austritt des Hauptstrahls für symmetrische Abbildung schon 1934 von Stephens ${ }^{11}$ angegeben worden. Dieser Ausdruck wurde dann von Brüche und Scherzer ${ }^{12}$ auf den Fall unsymmetrischer Abbildung erweitert. 1937 hat Cartan ${ }^{13}$ einen allgemeinen Ausdruck für die Ausdehnung des ionenoptischen Bildes für ein geradlinig begrenztes homogenes Sektorfeld für beliebige Ein- und Austrittswinkel angegeben. Um die Formel anwenden zu können, hat er sie in eine Reihe entwickelt und sich zur Vereinfachung dabei auf senkrechten Austritt des Hauptstrahls beschränkt.

Der Öffnungsfehler allein wurde für beliebige Ein- und Austrittswinkel und für geradlinige und

7 A. L. Hughes u. V. Rojansky, Phys. Rev. 34, 284 [1929].

8 E. G. Johnson u. A. O. Nier, Phys. Rev. 91, 10 [1953].

9 H. G. Voorhies, Rev. Sci. Instrum. 26, 716 [1955]; 27, 58 [1956].

10 H. Marschall, Phys. Z. 45, 1 [1944].

11 W. E. Stephens, Phys. Rev. 45, 513 [1934].

12 L. Brüche u. O. Scherzer, Geometrische Elektronenoptik, Springer-Verlag, Berlin 1934.

13 L. Cartan, J. Phys. Radium 8, 453 [1937].

14 H. Hintenberger, Z. Naturforschg. 6 a, 275 [1951].

15 W. R. Smythe, L. H. Rumbaugh u. S. S. West, Phys. Rev. 45, 724 [1934]; s. a. F. Bernhard, Z. angew. Phys. 9, 68 [1957].

16 a) H. Hintenberger, Z. Naturforschg. 3 a, 225 [1948].

b) H. Hintenberger, Z. Naturforschg. 3 a, 669 [1948].

c) H. Hintenberger, Rev. Sci. Instrum. 20, 748 [1949].

17 L. Kerwin, Rev. Sci. Instrum. 20, 36 [1949]; 21, 97 [1950]. - L. Kerwin u. Cl. Geoffrion, Rev. Sci. Instrum. 20, 381 [1949].

18 J. Geerk, Phys. Verh. 1950, S. 100. - J. Geerk u. C. Heinz, Z. Phys. 133, 513 [1952]. gekrümmte Magnetfeldgrenzen von dem einen von uns berechnet ${ }^{14}$. Daraus ergeben sich Bedingungen für die Korrektur des Öffnungsfehlers. Magnetfelder, bei denen der Öffnungsfehler korrigiert ist, sind von einer Reihe verschiedener Autoren angegeben worden ${ }^{15-21}$.

Für den Fall senkrechten Ein- und Austritts sind außer dem Öffnungsfehler auch noch die von $\alpha \beta$ und von $\beta^{2}$ abhängigen Bildfehler von Voorhies ${ }^{9}$ berechnet worden. Für beliebige Ein- und Austrittswinkel haben wir vor einiger Zeit diese 3 Bildfehler sowohl für homogene Magnetfelder mit geradlinigen $^{22}$ als auch mit gekrümmten Feldgrenzen ${ }^{23}$ bestimmt.

NIER und Mitarb. haben ein doppelfokussierendes Massenspektrometer mit hintereinandergeschaltetem elektrischem und magnetischem Ablenkfeld berechnet $^{8}$ und gebaut ${ }^{24,25}$, bei dem bei senkrechtem Einund Austritt des Hauptstrahls in beiden Feldern durch geeignete Wahl der Bild- und Gegenstandsweiten für eine Masse Doppelfokussierung erster Ordnung und Richtungsfokussierung zweiter Ordnung erreicht wird. Bedingungen, unter denen auch bei beliebigem Ein- und Austrittswinkel im Magnetfeld zusätzlich zur Doppelfokussierung erster Ordnung Richtungsfokussierung zweiter Ordnung erreicht werden kann, sind von dem einen von uns 1951 veröffentlicht worden ${ }^{26}$.

1955 haben wir zusammen mit Wende $27,28,29$ eine ausführliche Berechnung der Korrekturbedingungen der von $\alpha^{2}, \alpha \beta$ und $\beta^{2}$ abhängigen Bildfehler für Massenspektrographen, die Doppelfokussierung erster Ordnung für alle Massen zeigen, durchgeführt und die Bedingungen dafür angegeben, unter denen

19 C. Reuterswärd, Ark. Fys. 3, 53 [1951] ; 4, 159 [1954].

20 R. Persson, Ark. Fys. 3, 488 [1951].

21 E. T. S. Walton, Proc. R. Irish Acad. A 57, No. 1 [1954]

22 H. Hintenberger u. L. A. König, Z. Naturforschg. 11 a, 1039 [1956].

23 L. A. König u. H. Hintenberger, Z. Naturforschg. 12 a, 377 [1957].

24 A. O. Nier u. F. R. Roberts, Phys. Rev. 75, 386 [1949]; 81, 507 [1951].

25 A. O. Nier, Vortrag No. 16 in „Nuclear Masses and their Determination“ Pergamon Press London (1957) (Edited by H. Hintenberger).

26 H. Hintenberger, Mass Spectroscopy in Physics Research. Nat. Bur. Stand. Circ. 522, 95 [1953].

27 H. Hintenberger, H. Wende u. L. A. König, Z. Naturforschg 10 a, 344 [1955].

28 H. Hintenberger, H. Wende u. L. A. König, Z. Naturforschg 10 a, 605 [1955].

29 H. Hintenberger, H. Wende u. L. A. König, Z. Naturforschg 12 a, 88 [1957]. 
in solchen Instrumenten zusätzlich für eine Masse Richtungsfokussierung zweiter Ordnung erreicht werden kann. Unter Umständen ist es möglich, noch einen der beiden anderen Bildfehler (Glieder mit $\alpha \beta$ oder $\beta^{2}$ ) zusätzlich zum Öffnungsfehler zu korrigieren. Es ist aber grundsätzlich nicht möglich, in solchen Instrumenten vollständige Doppelfokussierung zweiter Ordnung zu erreichen *

Kürzlich wurden von uns die von $\alpha^{2}, \alpha \beta$ und $\beta^{2}$ abhängigen Bildfehler ganz allgemein für Massenspektrographen und Massenspektrometer, die räumlich getrennte elektrische und magnetische Ablenkfelder aufweisen, berechnet und die Gleichungen für die Korrektur dieser Bildfehler angegeben ${ }^{30}$. Diese vorangegangene Arbeit stellt den Ausgangspunkt für die vorliegende Untersuchung dar.

Inzwischen haben EwaLd und Mitarb. ${ }^{31}$ unsere frühere Untersuchung ${ }^{27,28,} 29$ über die Bildfehler in doppelfokussierenden Massenspektrographen mit gerader Bildkurve auf solche Apparate ausgedehnt, in denen ein Toroidkondensator statt eines Zylinderkondensators als elektrisches Ablenkfeld verwendet wird und gezeigt, daß auch hier im wesentlichen die gleichen Möglichkeiten für die Korrektur der Bildfehler bestehen. In diesen Apparaten wird zusätzlich zu den Fokussierungseigenschaften in der Ablenkebene noch Richtungsfokussierung senkrecht zur Ablenkebene erreicht. Neuerdings ist sowohl von LiEBL und Ewald ${ }^{32,} 33$ als auch von Robinson ${ }^{34}$ der Einfluß der Krümmung der Magnetfeldgrenze auf der Eintrittsseite zur Korrektur der Bildfehler in solchen Massenspektrographen ausgenutzt worden.

Der Vollständigkeit halber sei noch erwähnt, daß in einem homogenen magnetischen Feld, das von einem dazu senkrecht gerichteten homogenen elektrischen Feld überlagert ist, nicht nur genäherte sondern theoretisch exakte Doppelfokussierung erreicht werden kann ${ }^{35}$. Es sind verschiedene Instru-

* Im Falle gleichsinniger Ablenkung ist uns in der Arbeit ${ }^{28}$ bei den Korrekturbedingungen ein inzwischen berichtigter Vorzeichenfehler beim Übergang von einer Darstellungsform zu einer anderen unterlaufen, der weitere Korrekturmöglichkeiten für die Bildfehler vortäuschte ${ }^{27,} 28,29$.

30 H. Hintenberger u. L. A. König, Z. Naturforschg. 12 a, 140 [1957].

31 H. Ewald, H. Liebl u. G. Sauermann, Vortrag No. 17 in "Nuclear Masses and their Determination“ Pergamon Press London 1957 (Edited by H. Hintenberger).

32 H. Liebl u. H. Ewald, Z. Naturforschg. 12 a, 538 [1957].

33 H. Liebl u. H. Ewald, Z. Naturforschg. 12 a, 541 [1957].

34 C. F. Robinson, Rev. Sci. Instrum., im Druck.

35 W. Bleakney u. J. A. Hipple, Phys. Rev. 53, 521 [1938]. mente dieser Art gebaut worden ${ }^{36,37,38}$. Bei diesen Instrumenten ist aber, wenn großes Auflösungsvermögen erreicht werden soll, ein recht ausgedehnter Magnet erforderlich, da das homogene elektrische Feld zwischen seinen Polschuhen untergebracht werden muß.

Bei allen obengenannten Arbeiten wurde die Wirkung der Streufelder, die durch geeignete Blenden merklich herabgesetzt werden kann, vernachlässigt. Bei doppelfokussierenden Instrumenten ist der Polschuhabstand in der Regel sehr klein gegen den Krümmungsradius der Ionenbahnen und außerdem ist die Länge der Spalte und Blenden senkrecht zur Ablenkebene stets klein im Vergleich zum Polschuhabstand. Wenn auch noch der Weg der Ionen in den Ablenkfeldern groß ist gegenüber der Ausdehnung der Streufelder (nicht zu kleine Ablenkwinkel), ist eine Vernachlässigung bzw. eine vereinfachte Korrektur der Wirkung der Streufelder durch eine effektive Feldlänge gerechtfertigt. In verschiedenen Arbeiten ${ }^{39-44}$ ist der Einfluß der Streufelder auf die Abbildungseigenschaften der Ablenkfelder quantitativ abgeschätzt worden.

\section{Die Korrekturgleichungen für die Bildfehler}

In der bereits erwähnten früheren Arbeit ${ }^{30}$ wurden die Bildfehler für Massenspektrometer und Massenspektrographen mit hintereinandergeschaltetem elektrischem und magnetischem Ablenkfeld angegeben. Hierbei war vorausgesetzt, daß der Nebenstrahl nach Durchlaufen des elektrischen Feldes sich in der Form darstellen läßt:

$$
\begin{aligned}
y_{\mathrm{e}}= & r_{\mathrm{e}}\left\{K_{1} \alpha_{\mathrm{e}}+K_{2} \beta+K_{11} \alpha_{\mathrm{e}}^{2}+K_{12} \alpha_{\mathrm{e}} \beta+K_{22} \beta^{2}\right\}(1) \\
& +x_{\mathrm{e}}\left\{L_{1} \alpha_{\mathrm{e}}+L_{2} \beta+L_{11} \alpha_{\mathrm{e}}^{2}+L_{12} \alpha_{\mathrm{e}} \beta+L_{22} \beta^{2}\right\} .
\end{aligned}
$$

Die Größen $K$ und $L$ sind Abkürzungen der folgen. den Form:

36 G. W. Monk, J. D. Graves u. J. L. Horton, Rev. Sci. Instrum. 18, 796 [1947].

37 G. W. Monk u. G. K. Werner, Rev. Sci. Instrum. 20, 93 [1949].

38 T. Mariner u. W. Bleakney, Rev. Sci. Instrum. 20, 297 [1949].

39 M. Cotte, Thèses Univ. Paris 1938, Ser. A. No. 1807, No. d'ordre 2673.

40 R. Herzog, Z. Phys. 97, 596 [1935].

41 N. D. Coggeshall u. M. Muskat, Phys. Rev. 66, 187 [1944]. - N.D. Coggeshall, J. Appl. Phys. 18, 855 [1947].

42 W. Ploch u. W. Walcher, Z. Phys. 127, 274 [1950].

43 L. A. König u. H. Hintenberger, Z. Naturforschg. 10 a, 877 [1955].

44 R. Herzog, Z. Naturforschg. 10 a, 887 [1955]. 


$$
\begin{aligned}
& K_{1}=\varkappa_{1 \mathrm{a}}+\varkappa_{1 \mathrm{~b}} \frac{l_{\mathrm{e}}^{\prime}}{r_{\mathrm{e}}}, \\
& K_{2}=\varkappa_{2 \mathrm{a}}, \\
& K_{11}=\varkappa_{11 \mathrm{a}}+\varkappa_{11 \mathrm{~b}} \frac{l_{\mathrm{e}}^{\prime}}{r_{\mathrm{e}}}+\varkappa_{11 \mathrm{c}}\left(\frac{l_{\mathrm{e}}^{\prime}}{r_{\mathrm{e}}}\right)^{2}, \\
& K_{12}=\varkappa_{12 \mathrm{a}}+\varkappa_{12 \mathrm{~b}} \frac{l_{\mathrm{e}}^{\prime}}{r_{\mathrm{e}}}, \\
& K_{22}=\varkappa_{22 \mathrm{a}} ; \\
& L_{1}=\lambda_{1 \mathrm{a}}+\lambda_{1 \mathrm{~b}} \frac{l_{\mathrm{e}}^{\prime}}{r_{\mathrm{e}}}, \\
& L_{2}=\lambda_{2 \mathrm{a}}, \quad l_{11 \mathrm{a}}+\lambda_{11 \mathrm{~b}} \frac{l_{\mathrm{e}}^{\prime}}{r_{\mathrm{e}}}+\lambda_{11 \mathrm{c}}\left(\frac{l_{\mathrm{e}}^{\prime}}{r_{\mathrm{e}}}\right)^{2}, \\
& L_{11}=\lambda_{12}=\lambda_{12 \mathrm{a}}+\lambda_{12 \mathrm{~b}} \frac{l_{\mathrm{e}}^{\prime}}{r_{\mathrm{e}}}, \\
& L_{22}=\lambda_{22 \mathrm{a}}, \quad
\end{aligned}
$$

wobei die Größen $\varkappa$ und $\lambda$ von der Art und den Dimensionen des elektrischen Feldes abhängen. Für den Fall eines elektrischen Zylinderkondensators werden diese Größen später als Gln. (11) und (12) im Detail angegeben. Koordinatensystem und Bezeichnungen sind in Abb. 1 erklärt.

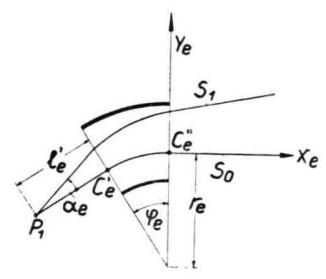

Abb. 1. Koordinatensystem zur Bahnberechnung des aus dem Zylinderkondensator austretenden Ionenstrahls $\left(s_{0}=\right.$ Mittelbahn, die von Teilchen der Geschwindigkeit $v=v_{0}$ mit $\alpha_{\mathrm{e}}=0$ durchlaufen wird; $s_{1}=$ Nachbarbahn von Teilchen mit $v=v_{0}(1+\beta)$ und $\left.\alpha_{\mathrm{e}} \neq 0\right)$.

Eine analoge Voraussetzung wurde für den aus dem Magnetfeld austretenden Nebenstrahl gemacht:

$y_{\mathrm{m}}=r_{\mathrm{m}}\left\{M_{1} \alpha_{\mathrm{m}}+M_{2} \beta+M_{11} \alpha_{\mathrm{m}}^{2}+M_{12} \alpha_{\mathrm{m}} \beta+M_{22} \beta^{2}\right\}$

$$
+x_{\mathrm{m}}\left\{N_{1} \alpha_{\mathrm{m}}+N_{2} \beta+N_{11} \alpha_{\mathrm{m}}^{2}+N_{12} \alpha_{\mathrm{m}} \beta+N_{22} \beta^{2}\right\} .
$$

Koordinatensystem und Bezeichnungen sind in Abb. 2 erklärt. Über die Größen $M$ und $N$ ist zusätzlich vorausgesetzt, daß sie sich in der Form

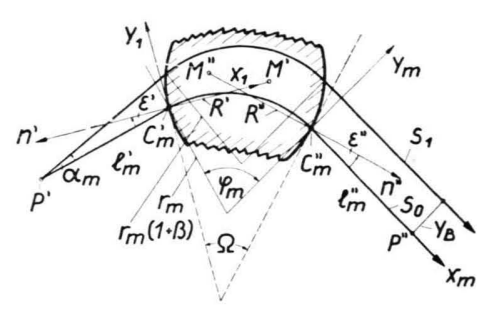

Abb. 2. Die Koordinatensysteme $x_{\mathrm{m}}, y_{\mathrm{m}}$ und $x_{1}, y_{1}$, die zur Bahnberechnung für das homogene Sektorfeld benutzt wurden $\left(s_{0}=\right.$ Mittelbahn, die von Teilchen der Geschwindigkeit $v=v_{0}$ mit $a_{\mathrm{m}}=0$ durchlaufen wird; $s_{1}=$ Nachbarbahn von Teilchen mit $v=v_{0}(1+\beta)$ und $\left.\alpha_{\mathrm{m}} \neq 0\right)$. $\varepsilon^{\prime}$ und $\varepsilon^{\prime \prime}$ sind positiv, wenn die Normalen $n^{\prime}$ und $n^{\prime \prime}$ auf der vom Ablenkungszentrum abgewandten Seite von $s_{0}$ liegen. Die Krümmungsradien der Feldgrenzen $R^{\prime}$ und $R^{\prime \prime}$ in den Punkten $C_{\mathrm{m}}^{\prime}$ und $C_{\mathrm{m}}{ }^{\prime \prime}$ sind für konvexe Krümmung der Polschuhe (wie in der Abb.) positiv, für konkave Krümmung negativ in die angegebenen Formeln einzusetzen.

$$
\begin{aligned}
& M_{1}=\mu_{1 \mathrm{a}}+\mu_{1 \mathrm{~b}} \frac{l_{\mathrm{m}}^{\prime}}{r_{\mathrm{m}}}, \\
& M_{2}=\mu_{2 \mathrm{a}}, \\
& M_{11}=\mu_{11 \mathrm{a}}+\mu_{11 \mathrm{~b}} \frac{l_{\mathrm{m}}^{\prime}}{r_{\mathrm{m}}}+\mu_{11 \mathrm{c}}\left(\frac{l_{\mathrm{m}}^{\prime}}{r_{\mathrm{m}}}\right)^{2}, \\
& M_{12}=\mu_{12 \mathrm{a}}+\mu_{12 \mathrm{~b}} \frac{l_{\mathrm{m}}^{\prime}}{r_{\mathrm{m}}}, \\
& M_{22}=\mu_{22 \mathrm{a}} ; \\
& N_{1}=v_{1 \mathrm{a}}+v_{1 \mathrm{~b}} \frac{l_{\mathrm{m}}^{\prime}}{r_{\mathrm{m}}}, \\
& N_{2}=v_{2 \mathrm{a}}, \quad\left(\frac{l_{\mathrm{m}}^{\prime}}{r_{\mathrm{m}}}\right)^{2}, \\
& N_{11}=v_{11 \mathrm{a}}+v_{11 \mathrm{~b}} \frac{l_{\mathrm{m}}^{\prime}}{r_{\mathrm{m}}}+v_{11 \mathrm{~s}} \\
& N_{12}=v_{12 \mathrm{a}}+v_{12 \mathrm{~b}} \frac{l_{\mathrm{m}}^{\prime}}{r_{\mathrm{m}}}, \\
& N_{22}=v_{22 \mathrm{a}}
\end{aligned}
$$

zerlegen lassen, was zum Beispiel für gekrümmt begrenzte $^{23}$ und damit auch für geradlinig begrenzte ${ }^{22}$ homogene magnetische Sektorfelder der Fall ist ${ }^{45}$. Die Größen $\mu$ und $v$ sind von der Art und den Dimensionen des magnetischen Ablenkfeldes und auch vom Verlauf der Hauptbahn in diesem Feld abhängig. Für homogene magnetische Sektorfelder mit beliebig gekrümmten Feldgrenzen und beliebigen Ablenk- sowie Ein- und Austrittswinkeln sind

${ }^{45} \mathrm{Da}$ sich die $K, L$ bzw. die $M, N$ in gleicher Weise als Polynome in $l_{\mathrm{e}}^{\prime} / r_{\mathrm{e}}$ bzw. $l_{\mathrm{m}}^{\prime} / r_{\mathrm{m}}$ schreiben lassen, folgt ganz allgemein aus dem Potenzreihenansatz für $y_{\mathrm{e}}$ bzw. $y_{\mathrm{m}}$. 
diese Größen in den Gln. (13) und (14) im Detail angegeben.

Unter diesen Voraussetzungen für die Einzelfelder ergibt sich in einer Kombination zweier Felder (siehe Abb. 3 und Abb.4) für die Ordinate $y_{\mathrm{B}}$ des Punktes, in dem der Nebenstrahl auf einem zum Hauptstrahl senkrechten und durch den Punkt $x_{\mathrm{m}}=l_{\mathrm{m}}^{\prime \prime}$ der $x_{\mathrm{m}}$-Achse gehenden Schirm auftrifft, die Beziehung

$$
y_{\mathrm{B}}=r_{\mathrm{m}}\left\{B_{1} \alpha+B_{2} \beta+B_{11} \alpha^{2}+B_{12} \alpha \beta+B_{22} \beta^{2}\right\},
$$

worin die Größen $B$ Abkürzungen für die folgenden Ausdrücke sind:

$$
\begin{aligned}
B_{1}= & \pm S_{1 \mathrm{a}} L_{1} \pm S_{1 \mathrm{~b}} T_{1}, \\
B_{2}= & \pm S_{1 \mathrm{a}} L_{2} \pm S_{1 \mathrm{~b}} T_{2}+S_{2 \mathrm{a}}, \\
B_{11}= & \pm S_{1 \mathrm{a}} L_{11} \pm S_{1 \mathrm{~b}} T_{11}+S_{11 \mathrm{a}} L_{1}^{2}+S_{11 \mathrm{~b}} L_{1} T_{1}+S_{11 \mathrm{c}} T_{1}{ }^{2} \\
B_{12}= & \pm S_{1 \mathrm{a}} L_{12} \pm S_{1 \mathrm{~b}} T_{12}+2 S_{11 \mathrm{a}} L_{1} L_{2}+S_{11 \mathrm{~b}}\left(L_{1} T_{2}+L_{2} T_{1}\right) \\
& +2 S_{11 \mathrm{c}} T_{1} T_{2} \pm S_{12 \mathrm{a}} L_{1} \pm S_{12 \mathrm{~b}} T_{1} \\
B_{22}= & \pm S_{1 \mathrm{a}} L_{22} \pm S_{1 \mathrm{~b}} T_{22}+S_{11 \mathrm{a}} L_{2}{ }^{2}+S_{11 \mathrm{~b}} L_{2} T_{2}+S_{11 \mathrm{c}} T_{2}{ }^{2} \\
& \pm S_{12 \mathrm{a}} L_{2} \pm S_{12 \mathrm{~b}} T_{2}+S_{22 \mathrm{a}} .
\end{aligned}
$$

(Das obere Vorzeichen gilt für gleichsinnige Ablenkung, das untere für gegensinnige Ablenkung im elektrischen und magnetischen Feld.)

Die Größen $S_{i}$ und $T_{k}$ haben die Bedeutung:

$$
\begin{gathered}
S_{i}=\mu_{i}+v_{i} \frac{l_{\mathrm{m}}^{\prime \prime}}{r_{\mathrm{m}}} \quad(i=1 \mathrm{a}, 1 \mathrm{~b}, 2 \mathrm{a}, 11 \mathrm{a}, 11 \mathrm{~b}, 11 \mathrm{c}, 12 \mathrm{a}, 12 \mathrm{~b}, 22 \mathrm{a}) \\
T_{k}=K_{k} \frac{r_{\mathrm{e}}}{r_{\mathrm{m}}}+L_{k} \frac{d}{r_{\mathrm{m}}}(k=1,2,11,12,22) .
\end{gathered}
$$

Die Ausdrücke $K$ und $L$ sind durch die Gln. (2) und (3) gegeben, die Größen $\mu$ und $\nu$ wurden durch die Gln. (5) und (6) definiert. $d$ ist der Abstand der beiden Felder (siehe Abb. 3 und Abb. 4). $B_{1}=0$ ist die Bedingung für Richtungsfokussierung erster Ordnung. $B_{2}$ ist der Koeffizient der Geschwindigkeitsdispersion und daher $B_{2}=0$ die Bedingung für Geschwindigkeitsfokussierung erster Ordnung. $B_{11}, B_{12}$ und $B_{22}$ sind die Koeffizienten für den Öffnungsfehler, die gemischt geschwindigkeits- und

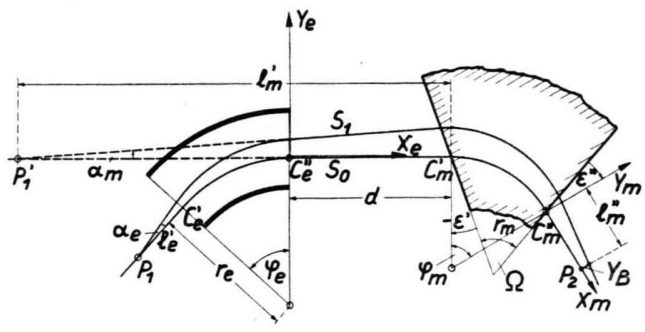

Abb. 3. Bahnverlauf (schematisch) bei gleichsinniger Ablenkung im elektrischen und magnetischen Feld. Die Lage des „Zwischenbildes" $P_{1}^{\prime}$ ist von $\alpha_{\mathrm{e}}$ und $\beta$ abhängig $\left(s_{0}=\right.$ Mittelbahn, die von Teilchen der Geschwindigkeit $v=v_{0}$ mit $\alpha_{\mathrm{e}}=0$ durchlaufen wird; $s_{1}=$ Nachbarbahn von Teilchen mit $v=v_{0}(1+\beta)$ und $\left.\alpha_{\mathrm{e}} \neq 0\right)$. öffnungswinkelabhängige Aberration und die rein geschwindigkeitsabhängige Aberration.

In einem doppelfokussierenden Massenspektrographen sind in der Regel ein elektrisches und ein magnetisches Feld hintereinandergeschaltet. Für den Fall, daß als elektrisches Feld ein Zylinderkondensator und als magnetisches Feld ein beliebig begrenztes homogenes magnetisches Sektorfeld benutzt wird, sind die in den Gln. (2) und (3) vorkommenden Koeffizienten $\varkappa$ und $\lambda$ bzw. die in den Gln. (5) und (6) vorkommenden Koeffizienten $\mu$ und $v$ (siehe Anm. ${ }^{23}$ ) gegeben durch

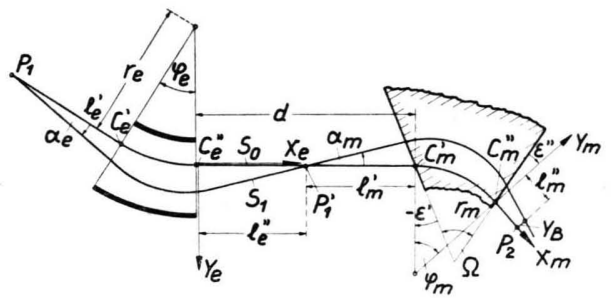

Abb. 4. Bahnverlauf (schematisch) bei gegensinniger Ablenkung im elektrischen und magnetischen Feld. Die Lage des "Zwischenbildes" $P_{1}^{\prime}$ ist von $\alpha_{\mathrm{e}}$ und $\beta$ abhängig ( $s_{0}=$ Mittelbahn, die von Teilchen der Geschwindigkeit $v=v_{0}$ mit $\alpha_{\mathrm{e}}=0$ durchlaufen wird; $s_{1}=$ Nachbarbahn von Teilchen mit $v=v_{0}(1+\beta)$ und $\left.\alpha_{\mathrm{e}} \neq 0\right)$. 
$\varkappa_{1 \mathrm{a}}=\frac{1}{\sqrt{2}} \sin \sqrt{ } 2 \varphi_{\mathrm{e}}$,

$\varkappa_{1 \mathrm{~b}}=\cos \sqrt{2} \varphi_{\mathrm{e}}$,

$\varkappa_{2 \mathrm{a}}=1-\cos \sqrt{2} \varphi_{\mathrm{e}}$,

$\varkappa_{11 \mathrm{a}}=-\frac{3}{8}+\frac{2}{3} \cos \sqrt{ } 2 \varphi_{\mathrm{e}}-\frac{7}{24} \cos 2 \sqrt{2} \varphi_{\mathrm{e}}$,

$\varkappa_{11 \mathrm{~b}}=-\left(\frac{4}{3 \sqrt{2}} \sin \sqrt{2} \varphi_{\mathrm{e}}-\frac{7}{6 / 2} \sin 2 \sqrt{2} \varphi_{\mathrm{e}}\right)$,

$\varkappa_{11 \mathrm{c}}=-\frac{3}{4}+\frac{1}{6} \cos \sqrt{2} \varphi_{\mathrm{e}}+\frac{7}{12} \cos 2 \sqrt{2} \varphi_{\mathrm{e}}$,

$\varkappa_{12 \mathrm{a}}=\frac{7}{3 \sqrt{2}}\left(\sin \sqrt{2} \varphi_{\mathrm{e}}-\frac{1}{2} \sin 2 \sqrt{2} \varphi_{\mathrm{e}}\right)$,

$x_{12 \mathrm{~b}}=\frac{3}{2}-\frac{1}{3} \cos \sqrt{2} \varphi_{\mathrm{e}}-\frac{7}{6} \cos 2 \sqrt{2} \varphi_{\mathrm{e}}$,

$\varkappa_{22 \mathrm{a}}=\frac{1}{4}-\frac{5}{6} \cos \sqrt{2} \varphi_{\mathrm{e}}+\frac{7}{12} \cos 2 \sqrt{2} \varphi_{\mathrm{e}} ;$

$$
\begin{aligned}
& \lambda_{1 \mathrm{a}}=\cos \sqrt{2} \varphi_{\mathrm{e}}, \\
& \lambda_{1 \mathrm{~b}}=-\sqrt{2} \sin \sqrt{2} \varphi_{\mathrm{e}}, \\
& \lambda_{2 \mathrm{a}}=\sqrt{2} \sin \sqrt{2} \varphi_{\mathrm{e}} \\
& \lambda_{11 \mathrm{a}}=-\frac{\sqrt{2}}{3}\left(2 \sin \sqrt{2} \varphi_{\mathrm{e}}-\sin 2 \sqrt{2} \varphi_{\mathrm{e}}\right), \\
& \lambda_{11 \mathrm{~b}}=-\frac{4}{3}\left(\cos \sqrt{2} \varphi_{\mathrm{e}}-\cos 2 \sqrt{2} \varphi_{\mathrm{e}}\right), \\
& \lambda_{11 \mathrm{c}}=-\frac{\sqrt{2}}{6}\left(\sin \sqrt{2} \varphi_{\mathrm{e}}+4 \sin 2 \sqrt{2} \varphi_{\mathrm{e}}\right), \\
& \lambda_{12 \mathrm{a}}=\frac{4}{3}\left(\cos \sqrt{2} \varphi_{\mathrm{e}}-\cos 2 \sqrt{2} \varphi_{\mathrm{e}}\right), \\
& \lambda_{12 \mathrm{~b}}=\frac{4}{3} \sqrt{2}\left(\sin \sqrt{2} \varphi_{\mathrm{e}}+\sin 2 \sqrt{2} \varphi_{\mathrm{e}}\right), \\
& \lambda_{22 \mathrm{a}}=-\frac{\sqrt{2}}{6}\left(\sin \sqrt{2} \varphi_{\mathrm{e}}+4 \sin 2 \sqrt{2} \varphi_{\mathrm{e}}\right)
\end{aligned}
$$

[Die Bedeutung der in (11) und (12) vorkommenden Größen ist aus Abb. 1 zu ersehen.]

$$
\begin{aligned}
& \mu_{1 \mathrm{a}}=\sin \varphi_{\mathrm{m}}, \\
& \mu_{1 \mathrm{~b}}=\frac{\cos \left(\varphi_{\mathrm{m}}-\varepsilon^{\prime}\right)}{\cos \varepsilon^{\prime}}, \\
& \mu_{2 \mathrm{a}}=1-\cos \varphi_{\mathrm{m}}, \\
& \mu_{11 \mathrm{a}}=\frac{1}{2}\left[\cos \varphi_{\mathrm{m}}\left(1-\cos \varphi_{\mathrm{m}}\right)+\operatorname{tg}^{2} \varepsilon^{\prime \prime} \sin ^{2} \varphi_{\mathrm{m}}\right], \\
& \mu_{11 \mathrm{~b}}=\frac{\cos \left(\varphi_{\mathrm{m}}-\varepsilon^{\prime}\right)}{\cos \varepsilon^{\prime}}\left[\operatorname{tg} \varepsilon^{\prime}+\operatorname{tg} \varepsilon^{\prime \prime} \frac{\cos \left(\varphi_{\mathrm{m}}-\varepsilon^{\prime \prime}\right)}{\cos \varepsilon^{\prime \prime}}\right]+\cos \varphi_{\mathrm{m}} \frac{\sin \left(\varphi_{\mathrm{m}}-\varepsilon^{\prime}-\varepsilon^{\prime \prime}\right)}{\cos \varepsilon^{\prime} \cos \varepsilon^{\prime \prime}}, \\
& \mu_{11 \mathrm{c}}=-\frac{1}{2} \frac{\sin \left(\varphi_{\mathrm{m}}-\varepsilon^{\prime}-\varepsilon^{\prime \prime}\right)}{\cos \varepsilon^{\prime} \cos \varepsilon^{\prime \prime}}\left[\frac{\sin \left(\varphi_{\mathrm{m}}-\varepsilon^{\prime}-\varepsilon^{\prime \prime}\right)}{\cos \varepsilon^{\prime} \cos \varepsilon^{\prime \prime}}+2 \operatorname{tg} \varepsilon^{\prime \prime} \frac{\cos \left(\varphi_{\mathrm{m}}-\varepsilon^{\prime}\right)}{\cos \varepsilon^{\prime}}\right]+\frac{r_{\mathrm{m}}}{2 R^{\prime} \cos ^{3} \varepsilon^{\prime}} \sin \varphi_{\mathrm{m}}, \\
& \mu_{12 \mathrm{a}}=\sin \varphi_{\mathrm{m}} \frac{1-\cos \varphi_{\mathrm{m}}}{\cos ^{2} \varepsilon^{\prime \prime}}, \\
& \mu_{12 \mathrm{~b}}=\operatorname{tg} \varepsilon^{\prime \prime}\left[\operatorname{tg} \varepsilon^{\prime \prime}+\frac{\sin \left(\varphi_{\mathrm{m}}-\varepsilon^{\prime \prime}\right)}{\cos \varepsilon^{\prime \prime}}\right] \frac{\cos \left(\varphi_{\mathrm{m}}-\varepsilon^{\prime}\right)}{\cos \varepsilon^{\prime}}+\sin \varphi_{\mathrm{m}} \frac{\sin \left(\varphi_{\mathrm{m}}-\varepsilon^{\prime}-\varepsilon^{\prime \prime}\right)}{\cos \varepsilon^{\prime} \cos \varepsilon^{\prime \prime}}, \\
& \mu_{22 \mathrm{a}}=\operatorname{tg} \varepsilon^{\prime \prime}\left[\operatorname{tg} \varepsilon^{\prime \prime}+\frac{\sin \left(\varphi_{\mathrm{m}}-\varepsilon^{\prime \prime}\right)}{\cos \varepsilon^{\prime \prime}}\right]\left(1-\cos \varphi_{\mathrm{m}}\right)-\frac{1}{2}\left[\operatorname{tg} \varepsilon^{\prime \prime}+\frac{\sin \left(\varphi_{\mathrm{m}}-\varepsilon^{\prime \prime}\right)}{\cos \varepsilon^{\prime \prime}}\right]^{2} ; \\
& v_{1 \mathrm{a}}=\frac{\cos \left(\varphi_{\mathrm{m}}-\varepsilon^{\prime \prime}\right)}{\cos \varepsilon^{\prime \prime}}, \\
& v_{1 \mathrm{~b}}=-\frac{\sin \left(\varphi_{\mathrm{m}}-\varepsilon^{\prime}-\varepsilon^{\prime \prime}\right)}{\cos \varepsilon^{\prime} \cos \varepsilon^{\prime \prime}}, \\
& \nu_{2 \mathrm{a}}=\operatorname{tg} \varepsilon^{\prime \prime}+\frac{\sin \left(\varphi_{\mathrm{m}}-\varepsilon^{\prime \prime}\right)}{\cos \varepsilon^{\prime \prime}}, \\
& v_{11 \mathrm{a}}=-\frac{1}{2}\left[\frac{\sin \left(\varphi_{\mathrm{m}}-\varepsilon^{\prime \prime}\right)}{\cos \varepsilon^{\prime \prime}}+\operatorname{tg} \varepsilon^{\prime \prime} \frac{\cos ^{2}\left(\varphi_{\mathrm{m}}-\varepsilon^{\prime \prime}\right)}{\cos ^{2} \varepsilon^{\prime \prime}}\right]+\frac{r_{\mathrm{m}}}{2 R^{\prime \prime} \cos ^{3} \varepsilon^{\prime \prime}} \sin ^{2} \varphi_{\mathrm{m}}, \\
& v_{11 \mathrm{~b}}=-\frac{\sin \left(\varphi_{\mathrm{m}}-\varepsilon^{\prime}-\varepsilon^{\prime \prime}\right)}{\cos \varepsilon^{\prime} \cos \varepsilon^{\prime \prime}}\left[\operatorname{tg} \varepsilon^{\prime}-\operatorname{tg} \varepsilon^{\prime \prime} \frac{\cos \left(\varphi_{\mathrm{m}}-\varepsilon^{\prime \prime}\right)}{\cos \varepsilon^{\prime \prime}}\right]+\frac{r_{\mathrm{m}}}{R^{\prime \prime} \cos ^{3} \varepsilon^{\prime \prime}} \sin \varphi_{\mathrm{m}} \frac{\cos \left(\varphi_{\mathrm{m}}-\varepsilon^{\prime}\right)}{\cos \varepsilon^{\prime}}, \\
& v_{11 \mathrm{c}}=-\frac{1}{2} \operatorname{tg} \varepsilon^{\prime \prime} \frac{\sin ^{2}\left(\varphi_{\mathrm{m}}-\varepsilon^{\prime}-\varepsilon^{\prime \prime}\right)}{\cos ^{2} \varepsilon^{\prime} \cos ^{2} \varepsilon^{\prime \prime}}+\frac{r_{\mathrm{m}}}{2 R^{\prime \prime} \cos ^{3} \varepsilon^{\prime \prime}} \frac{\cos ^{2}\left(\varphi_{\mathrm{m}}-\varepsilon^{\prime}\right)}{\cos ^{2} \varepsilon^{\prime}}+\frac{r_{\mathrm{m}}}{2 R^{\prime} \cos ^{3} \varepsilon^{\prime}} \frac{\cos \left(\varphi_{\mathrm{m}}-\varepsilon^{\prime \prime}\right)}{\cos \varepsilon^{\prime \prime}},
\end{aligned}
$$




$$
\begin{aligned}
& v_{12 \mathrm{a}}=-\frac{\cos \left(\varphi_{\mathrm{m}}-\varepsilon^{\prime \prime}\right)}{\cos \varepsilon^{\prime \prime}}\left[\operatorname{tg} \varepsilon^{\prime \prime}+\frac{\sin \left(\varphi_{\mathrm{m}}-\varepsilon^{\prime \prime}\right)}{\cos \varepsilon^{\prime \prime}}\right] \operatorname{tg} \varepsilon^{\prime \prime}+\frac{r_{\mathrm{m}}}{R^{\prime \prime} \cos ^{3} \varepsilon^{\prime \prime}}\left(1-\cos \varphi_{\mathrm{m}}\right) \sin \varphi_{\mathrm{m}}, \\
& v_{12 \mathrm{~b}}=\frac{\sin \left(\varphi_{\mathrm{m}}-\varepsilon^{\prime}-\varepsilon^{\prime \prime}\right)}{\cos \varepsilon^{\prime} \cos \varepsilon^{\prime \prime}}\left\{1+\operatorname{tg} \varepsilon^{\prime \prime}\left[\operatorname{tg} \varepsilon^{\prime \prime}+\frac{\sin \left(\varphi_{\mathrm{m}}-\varepsilon^{\prime \prime}\right)}{\cos \varepsilon^{\prime \prime}}\right]\right\}+\frac{r_{\mathrm{m}}}{R^{\prime \prime} \cos { }^{3} \varepsilon^{\prime \prime}} \frac{\cos \left(\varphi_{\mathrm{m}}-\varepsilon^{\prime}\right)}{\cos \varepsilon^{\prime}}\left(1-\cos \varphi_{\mathrm{m}}\right), \\
& v_{22 \mathrm{a}}=-\frac{1}{2} \operatorname{tg} \varepsilon^{\prime \prime}\left[\operatorname{tg} \varepsilon^{\prime \prime}+\frac{\sin \left(\varphi_{\mathrm{m}}-\varepsilon^{\prime \prime}\right)}{\cos \varepsilon^{\prime \prime}}\right]^{2}-\left[\operatorname{tg} \varepsilon^{\prime \prime}+\frac{\sin \left(\varphi_{\mathrm{m}}-\varepsilon^{\prime \prime}\right)}{\cos \varepsilon^{\prime \prime}}\right]+\frac{r_{\mathrm{m}}}{2 R^{\prime \prime} \cos ^{3} \varepsilon^{\prime \prime}}\left(1-\cos \varphi_{\mathrm{m}}\right)^{2} .
\end{aligned}
$$

[Die Bedeutung der in (13) und (14) vorkommenden Größen ist aus Abb. 2 zu ersehen. Die numerischen Rechnungen dieser Arbeit wurden für gerade Feldgrenzen, d. h. für $1 / R^{\prime}=1 / R^{\prime \prime}=0$ durchgeführt.]

Soll Doppelfokussierung erster Ordnung erreicht werden, so müssen die nachstehenden Gln. (15 a) und (15 b) erfüllt sein. Soll vollständige Doppelfokussierung zweiter Ordnung erreicht werden, so müssen die Gln. (15 a) bis (15 e) gleichzeitig erfüllt sein. Diese Gleichungen lauten:
a) $B_{1}=0$
c) $B_{11}=0$,
b) $B_{2}=0$,
d) $B_{12}=0$,
e) $B_{22}=0$.

Die Größen $B$ sind durch die Gln. (2), (3), (5), (6) und (8) bis (14) bestimmt.

\section{Die Lösung der Bedingungsgleichungen für die Bildfehlerkorrektur}

\section{Umformung des Gleichungssystems}

Die Gln. (15) stellen ein nichtlineares System von fünf Gleichungen mit acht Unbekannten $\varphi_{\mathrm{m}}, \varepsilon^{\prime}, \varepsilon^{\prime \prime}$, $\sqrt{2} \varphi_{\mathrm{e}}, r_{\mathrm{e}} / r_{\mathrm{m}}, d / r_{\mathrm{m}}, l_{\mathrm{e}}^{\prime} / r_{\mathrm{m}}, l_{\mathrm{m}}^{\prime \prime} / r_{\mathrm{m}}$ dar, zu dessen Lösung keine Ausgangsnäherungen zur Verfügung stehen.

Man erhält aus Gl. (15 b) unter Berücksichtigung der Gln. (8), (9) und (10) zusammen mit den Gln. (2) und (3)

$$
\frac{l_{\mathrm{m}}^{\prime \prime}}{r_{\mathrm{m}}}=-\frac{a_{\mathrm{z}}+b_{\mathrm{z}} \frac{r_{\mathrm{e}}}{r_{\mathrm{m}}}+c_{\mathrm{z}} \frac{d}{r_{\mathrm{m}}}}{a_{\mathrm{n}}+b_{\mathrm{n}} \frac{r_{\mathrm{e}}}{r_{\mathrm{m}}}+c_{\mathrm{n}} \frac{d}{r_{\mathrm{m}}}}
$$

$$
\begin{aligned}
& a_{\mathrm{z}}= \pm \lambda_{2 \mathrm{a}} \mu_{1 \mathrm{a}}+\mu_{2 \mathrm{a}}, \\
& b_{\mathrm{z}}= \pm \varkappa_{2 \mathrm{a}} \mu_{1 \mathrm{~b}}, \\
& c_{\mathrm{z}}= \pm \lambda_{2 \mathrm{a}} \mu_{1 \mathrm{~b}}, \\
& a_{\mathrm{n}}= \pm \lambda_{2 \mathrm{a}} v_{1 \mathrm{a}}+\nu_{2 \mathrm{a}} \\
& b_{\mathrm{n}}= \pm \varkappa_{2 \mathrm{a}} v_{1 \mathrm{~b}}, \\
& c_{\mathrm{n}}= \pm \lambda_{2 \mathrm{a}} v_{1 \mathrm{~b}} .
\end{aligned}
$$

Das obere Vorzeichen gilt für gleichsinnige, das untere für gegensinnige Ablenkung in den beiden Feldern.

Aus Gl. (15 a) ergibt sich unter Beachtung der Gln. (8), (9), (10), (2), (3), (16), (17) und (18)

$$
\frac{l_{\mathrm{e}}}{r_{\mathrm{e}}}=-\frac{A_{\mathrm{z}}+B_{\mathrm{z}} \frac{r_{\mathrm{e}}}{r_{\mathrm{m}}}+C_{\mathrm{z}} \frac{d}{r_{\mathrm{m}}}}{A_{\mathrm{n}}+B_{\mathrm{n}} \frac{r_{\mathrm{e}}}{r_{\mathrm{m}}}+C_{\mathrm{n}} \frac{d}{r_{\mathrm{m}}}}
$$

mit

$$
\begin{aligned}
& A_{\mathrm{z}}= \pm \lambda_{1 \mathrm{a}}\left(\mu_{1 \mathrm{a}} \nu_{2 \mathrm{a}}-\mu_{2 \mathrm{a}} v_{1 \mathrm{a}}\right) \\
& B_{\mathrm{z}}= \pm \varkappa_{1 \mathrm{a}}\left(\mu_{1 \mathrm{~b}} \nu_{2 \mathrm{a}}-\mu_{2 \mathrm{a}} v_{1 \mathrm{~b}}\right) \\
& \quad+\varkappa_{1 \mathrm{a}} \lambda_{2 \mathrm{a}}-\varkappa_{2 \mathrm{a}} \lambda_{1 \mathrm{a}} \\
& C_{\mathrm{z}}= \pm \lambda_{1 \mathrm{a}}\left(\mu_{1 \mathrm{~b}} \nu_{2 \mathrm{a}}-\mu_{2 \mathrm{a}} v_{1 \mathrm{~b}}\right) \\
& A_{\mathrm{n}}= \pm \lambda_{1 \mathrm{~b}}\left(\mu_{1 \mathrm{a}} \nu_{2 \mathrm{a}}-\mu_{2 \mathrm{a}} v_{1 \mathrm{a}}\right) \\
& B_{\mathrm{n}}= \pm \varkappa_{1 \mathrm{~b}}\left(\mu_{1 \mathrm{~b}} \nu_{2 \mathrm{a}}-\mu_{2 \mathrm{a}} \nu_{1 \mathrm{~b}}\right) \\
& \quad+\varkappa_{1 \mathrm{~b}} \lambda_{2 \mathrm{a}}-\varkappa_{2 \mathrm{a}} \lambda_{1 \mathrm{~b}} \\
& C_{\mathrm{n}}= \pm \lambda_{1 \mathrm{~b}}\left(\mu_{1 \mathrm{~b}} \nu_{2 \mathrm{a}}-\mu_{2 \mathrm{a}} v_{1 \mathrm{~b}}\right) .
\end{aligned}
$$

Aus Gl. (15 e) läßt sich unter Berücksichtigung der Gln. (8), (9), (10), (2), (3) und (16) und (17) $r_{\mathrm{e}} / r_{\mathrm{m}}$ ausrechnen. Man erhält dafür die Gleichung:

$$
\left(\frac{r_{\mathrm{e}}}{r_{\mathrm{m}}}\right)^{3}+a\left(\frac{r_{\mathrm{e}}}{r_{\mathrm{m}}}\right)^{2}+b\left(\frac{r_{\mathrm{e}}}{r_{\mathrm{m}}}\right)+c=0
$$

mit den Abkürzungen

$$
\begin{aligned}
& a=\frac{1}{b_{\mathrm{n}} R_{\mathrm{z}}-b_{\mathrm{z}} R_{\mathrm{n}}}\left\{\begin{array}{l}
\left.\left[\left(a_{\mathrm{n}} R_{\mathrm{z}}-a_{\mathrm{z}} R_{\mathrm{n}}\right)+\left(b_{\mathrm{n}} P_{\mathrm{z}}-b_{\mathrm{z}} P_{\mathrm{n}}\right)\right]+\left[\left(b_{\mathrm{n}} U_{\mathrm{z}}-b_{\mathrm{z}} U_{\mathrm{n}}\right)+\left(c_{\mathrm{n}} R_{\mathrm{z}}-c_{\mathrm{z}} R_{\mathrm{n}}\right)\right] \frac{d}{r_{\mathrm{m}}}\right\}, \\
b=\frac{1}{b_{\mathrm{n}} R_{\mathrm{z}}-b_{\mathrm{z}} R_{\mathrm{n}}}\left\{\begin{array}{c}
{\left[\left(a_{\mathrm{n}} P_{\mathrm{z}}-a_{\mathrm{z}} P_{\mathrm{n}}\right)+\left(b_{\mathrm{n}} O_{\mathrm{z}}-b_{\mathrm{z}} O_{\mathrm{n}}\right)\right]+\left[\left(a_{\mathrm{n}} U_{\mathrm{z}}-a_{\mathrm{z}} U_{\mathrm{n}}\right)+\left(b_{\mathrm{n}} Q_{\mathrm{z}}-b_{\mathrm{z}} Q_{\mathrm{n}}\right)\right.} \\
\left.+\left(c_{\mathrm{n}} P_{\mathrm{z}}-c_{\mathrm{z}} P_{\mathrm{n}}\right)\right] \frac{d}{r_{\mathrm{m}}}+\left[\left(b_{\mathrm{n}} V_{\mathrm{z}}-b_{\mathrm{z}} V_{\mathrm{n}}\right)+\left(c_{\mathrm{n}} U_{\mathrm{z}}-c_{\mathrm{z}} U_{\mathrm{n}}\right)\right]\left(\frac{d}{r_{\mathrm{m}}}\right)^{2}
\end{array}\right\}, \\
c=\frac{1}{b_{\mathrm{n}} R_{\mathrm{z}}-b_{\mathrm{z}} R_{\mathrm{n}}}\left\{\begin{array}{l}
{\left[a_{\mathrm{n}} O_{\mathrm{z}}-a_{\mathrm{z}} O_{\mathrm{n}}\right]+\left[\left(a_{\mathrm{n}} Q_{\mathrm{z}}-a_{\mathrm{z}} Q_{\mathrm{n}}\right)+\left(c_{\mathrm{n}} O_{\mathrm{z}}-c_{\mathrm{z}} O_{\mathrm{n}}\right)\right] \frac{d}{r_{\mathrm{m}}}} \\
+\left[\left(a_{\mathrm{n}} V_{\mathrm{z}}-a_{\mathrm{z}} V_{\mathrm{n}}\right)+\left(c_{\mathrm{n}} Q_{\mathrm{z}}-c_{\mathrm{z}} Q_{\mathrm{n}}\right)\right]\left(\frac{d}{r_{\mathrm{m}}}\right)^{2}+\left[c_{\mathrm{n}} V_{\mathrm{z}}-c_{\mathrm{z}} V_{\mathrm{n}}\right]\left(\frac{d}{r_{\mathrm{m}}}\right)^{3}
\end{array}\right\},
\end{array}\right.
\end{aligned}
$$


wobei die Größen $O, P, Q, R, U$ und $V$ definiert sind durch:

$$
\begin{aligned}
& O_{\mathrm{z}}= \pm \lambda_{22 \mathrm{a}} \mu_{1 \mathrm{a}}+\lambda_{2 \mathrm{a}}^{2} \mu_{11 \mathrm{a}} \pm \lambda_{2 \mathrm{a}} \mu_{12 \mathrm{a}}+\mu_{22 \mathrm{a}} \text {, } \\
& P_{\mathrm{z}}= \pm \varkappa_{22 \mathrm{a}} \mu_{1 \mathrm{~b}}+\varkappa_{2 \mathrm{a}} \lambda_{2 \mathrm{a}} \mu_{11 \mathrm{~b}} \pm \varkappa_{2 \mathrm{a}} \mu_{12 \mathrm{~b}} \text {, } \\
& Q_{\mathrm{z}}= \pm \lambda_{22 \mathrm{a}} \mu_{1 \mathrm{~b}}+\lambda_{2 \mathrm{a}}^{2} \mu_{11 \mathrm{~b}} \pm \lambda_{2 \mathrm{a}} \mu_{12 \mathrm{~b}}, \\
& R_{\mathrm{z}}=x_{2 \mathrm{a}}^{2} \mu_{11 \mathrm{c}} \text {, } \\
& U_{\mathrm{z}}=2 \varkappa_{2 \mathrm{a}} \lambda_{2 \mathrm{a}} \mu_{11 \mathrm{c}} \text {, } \\
& V_{\mathrm{z}}=i_{2 \mathrm{a}}^{2} \mu_{11 \mathrm{c}} \text {; } \\
& O_{\mathrm{n}}= \pm \lambda_{22 \mathrm{a}} v_{1 \mathrm{a}}+\lambda_{2 \mathrm{a}}^{2} \nu_{11 \mathrm{a}} \pm \lambda_{2 \mathrm{a}} v_{12 \mathrm{a}}+v_{22 \mathrm{a}} \text {, } \\
& P_{\mathrm{n}}= \pm \varkappa_{2 \mathrm{a} a} v_{1 \mathrm{~b}}+\varkappa_{2 \mathrm{a}} \lambda_{2 \mathrm{a}} \nu_{11 \mathrm{~b}} \pm \varkappa_{2 \mathrm{a}} v_{12 \mathrm{~b}} \text {, } \\
& Q_{\mathrm{n}}= \pm \lambda_{22 \mathrm{a}} v_{1 \mathrm{~b}}+\lambda_{2 \mathrm{a}}^{2} \nu_{11 \mathrm{~b}} \pm \lambda_{2 \mathrm{a}} v_{12 \mathrm{~b}} \text {, } \\
& R_{\mathrm{n}}=\varkappa_{2 \mathrm{a}}^{2} \nu_{11 \mathrm{c}} \text {, } \\
& U_{\mathrm{n}}=2 \varkappa_{2 \mathrm{a}} \lambda_{2 \mathrm{a}} v_{11 \mathrm{c}} \text {, } \\
& V_{\mathrm{n}}=\lambda_{2 \mathrm{a}}^{2} v_{11 \mathrm{c}} \text {. }
\end{aligned}
$$

Ist Gl. (22) erfüllt, so verschwindet der von $\beta^{2} \mathrm{ab}$ hängige Bildfehler $\left(B_{22}=0\right)$.

Aus Gl. (22) erhält man die drei Wurzeln $r_{\mathrm{e}} / r_{\mathrm{m}}$ nach bekannten Formeln. Setzt man bei der numerischen Rechnung eine derselben [Gl. (26e)] in die Gln. (16) und (19) ein, so hat man neben $r_{\mathrm{e}} / r_{\mathrm{m}}$ auch $l_{\mathrm{e}}^{\prime} / r_{\mathrm{m}}$ und $l_{\mathrm{m}}^{\prime \prime} / r_{\mathrm{m}}$ als Funktionen von $\sqrt{2} \varphi_{\mathrm{e}}$, $d / r_{\mathrm{m}}, \varphi_{\mathrm{m}}, \varepsilon^{\prime}$ und $\varepsilon^{\prime \prime}$ dargestellt:

$$
\begin{aligned}
& \text { b) } \frac{l_{\mathrm{e}}^{\prime}}{r_{\mathrm{m}}}=f_{1}\left(\sqrt{2} \varphi_{\mathrm{e}}, \frac{d}{r_{\mathrm{m}}}, \varphi_{\mathrm{m}}, \varepsilon^{\prime}, \varepsilon^{\prime \prime}\right), \\
& \text { a) } \frac{l_{\mathrm{m}}^{\prime \prime}}{r_{\mathrm{m}}}=f_{2}\left(\sqrt{2} \varphi_{\mathrm{e}}, \frac{d}{r_{\mathrm{m}}}, \varphi_{\mathrm{m}}, \varepsilon^{\prime}, \varepsilon^{\prime \prime}\right), \\
& \text { e) } \frac{r_{\mathrm{e}}}{r_{\mathrm{m}}}=f_{3}\left(\sqrt{2} \varphi_{\mathrm{e}}, \frac{d}{r_{\mathrm{m}}}, \varphi_{\mathrm{m}}, \varepsilon^{\prime}, \varepsilon^{\prime \prime}\right) .
\end{aligned}
$$

(Die Bezeichnungen a, b, .. deuten die Herkunft der Gleichungen an.)

Diese Werte sind in die Gln. (15c) und (15d) für die von $\alpha^{2}$ und $\alpha \beta$ abhängigen Bildfehler einzusetzen. Damit bleiben die beiden Gleichungen:

$$
\begin{aligned}
& \text { c) } B_{11}\left(\sqrt{2} \varphi_{\mathrm{e}}, \frac{d}{r_{\mathrm{m}}}, \varphi_{\mathrm{m}}, \varepsilon^{\prime}, \varepsilon^{\prime \prime}\right)=0 \\
& \text { d) } B_{12}\left(\sqrt{2} \varphi_{\mathrm{e}}, \frac{d}{r_{\mathrm{m}}}, \varphi_{\mathrm{m}}, \varepsilon^{\prime}, \varepsilon^{\prime \prime}\right)=0
\end{aligned}
$$

zu lösen. Gibt man 3 der 5 Veränderlichen $\varphi_{\mathrm{m}}, \varepsilon^{\prime}$, $\varepsilon^{\prime \prime}, \sqrt{2} \varphi_{\mathrm{e}}$ und $d / r_{\mathrm{m}}$, die in die Gln. (27) eingehen, willkürlich vor, so hat man nunmehr 2 Gleichungen mit 2 Unbekannten zu lösen. Diese Aufgabe ist we- sentlich einfacher als die Lösung der 5 Gln. (15), wie die Erfahrung bestätigt. Die Lösungen werden hierdurch Funktionen der vorgegebenen 3 Parameter. Es ist zweckmäßig, die für das Magnetfeld charakteristischen Größen $\varphi_{\mathrm{m}}, \varepsilon^{\prime}$ und $\varepsilon^{\prime \prime}$ vorzugeben, zumal auch der Radius $r_{\mathrm{m}}$ der Mittelbahn im Magnetfeld als Längeneinheit gewählt wurde.

\section{Das angewandte numerische Rechenverfahren ${ }^{46}$}

Nach Vorgabe der Winkel $\varphi_{\mathrm{m}}, \varepsilon^{\prime}$ und $\varepsilon^{\prime \prime}$ wurden in $\operatorname{der}(\vartheta, \delta)$-Ebene $\left(\vartheta \equiv \sqrt{2} \varphi_{\mathrm{e}}, \delta \equiv d / r_{\mathrm{m}}\right)$ die Kurven (fortan Nullkurven genannt)

$$
\vartheta_{1}=\vartheta_{1}(\delta), \quad \vartheta_{2}=\vartheta_{2}(\delta)
$$

gesucht, für die nach den Gln. $(27 \mathrm{c})$ bzw. $(27 \mathrm{~d})$ unter Beachtung der Gln. (26) $B_{11}=0$ bzw. $B_{12}=0$ ist. Der Schnittpunkt der beiden Kurven ist eine gesuchte Lösung der Gln. (15), da auch $B_{1}=B_{2}=0$ ist, wenn die Gln. (26) beachtet werden.

Bei festem $\vartheta$ wurden für äquidistante Werte von $\delta$ im physikalisch vernünftigen Bereich $0 \leqq \delta \leqq 10 B_{11}$ und $B_{12}$ berechnet. Trat Vorzeichenwechsel einer dieser Größen ein, so wurde das Intervall, in dem der Vorzeichenwechsel auftrat, weiter unterteilt und auf diese Weise zu einem bestimmten $\vartheta$ der Wert von $\delta$ ermittelt, für den $B_{11}$ bzw. $B_{12}$ gleich Null (in der Praxis kleiner als $10^{-3}$ ) war. Zwei Beispiele für auf solchem Weg gewonnene Nullkurven sind in den Abb. 5 und 6 zu sehen. Um die

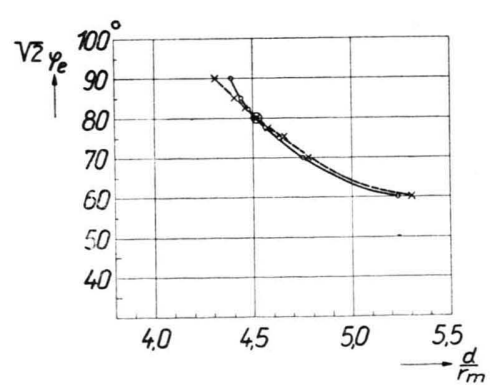

Abb. 5. Kurven, längs denen gleichzeitig Doppelfokussierung erster Ordnung herrscht und außerdem die Bildfehler $B_{11}=B_{22}=0 \quad(X)$ bzw. $B_{12}=B_{22}=0$ ( $\left.\bigcirc\right)$ sind, wenn $\varphi_{\mathrm{m}}=60^{\circ}, \varepsilon^{\prime}=0^{\circ}$ und $\varepsilon^{\prime \prime}=-15^{\circ}$ ist. Der Schnittpunkt beider Kurven ergibt die Werte von $\vartheta=\sqrt{2} \varphi_{\mathrm{e}}$ und $\delta=d / r_{\mathrm{m}}$ im Fall vollständiger Doppelfokussierung zweiter Ordnung $\left(B_{11}=\right.$ $\left.B_{12}=B_{22}=0\right)$ zu dem vorgegebenen Wertetripel $\left(\varphi_{\mathrm{m}}, \varepsilon^{\prime}, \varepsilon^{\prime \prime}\right)$. (Beispiel für eine Lösung aus Lösungssystem I für gleichsinnige Ablenkung, siehe Apparat Nr. 3 in Tab. 1).

46 Die numerische Rechnung wurde auf einem IBM-Magnettrommel-Rechner Type 650 im Rechenzentrum der Firma IBM Deutschland in Sindelfingen (Württ.) durchgeführt. 
Wertetripel $\left(\varphi_{\mathrm{m}}, \varepsilon^{\prime}, \varepsilon^{\prime \prime}\right)$ zu finden, für die sich physikalisch sinnvolle Lösungen ergeben, wurden zuvor für vorgegebene Wertesätze $\left(\varphi_{\mathrm{m}}, \varepsilon^{\prime}, \varepsilon^{\prime \prime}, \vartheta, \delta\right)$ die Werte von $r_{\mathrm{e}} / r_{\mathrm{m}}, l_{\mathrm{e}}^{\prime} / r_{\mathrm{m}}$ und $l_{\mathrm{m}}^{\prime \prime} / r_{\mathrm{m}}$ nach den Gln. (22), (19) und (16) berechnet. Diese Wertesätze wurden gleichmäßig in äquidistanten Abständen gewählt.

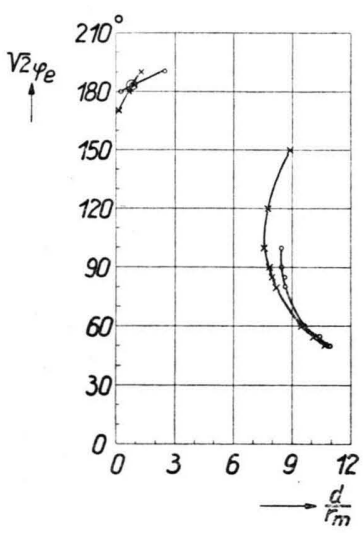

Abb. 6. Kurven, längs denen gleichzeitig Doppelfokussierung erster Ordnung herrscht und außerdem die Bildfehler $B_{11}=$ $B_{22}=0(X)$ bzw. $B_{12}=B_{22}=0(\bigcirc)$ sind für das Wertetripel $\varphi_{\mathrm{m}}=60^{\circ}, \varepsilon^{\prime}=15^{\circ}, \varepsilon^{\prime \prime}=-15^{\circ}$. Der Schnittpunkt beider Kurven ergibt die Werte von $\vartheta=\sqrt{2} \varphi_{\mathrm{e}}$ und $\delta=d / r_{\mathrm{m}}$ im Fall vollständiger Doppelfokussierung zweiter Ordnung $\left(B_{11}=B_{12}\right.$ $\left.=B_{22}=0\right) \mathrm{zu}$ dem vorgegebenen Wertetripel $\left(\varphi_{\mathrm{m}}, \varepsilon^{\prime}, \varepsilon^{\prime \prime}\right)$. An diesem Beispiel ist gegenüber dem der Abb. 5 besonders interessant, daß ein brauchbarer Schnittpunkt an einer ganz anderen Stelle liegt, als man bei der Durchführung der Rechnung zunächst vermutet. (Beispiel aus Lösungssystem II für gleichsinnige Ablenkung, siehe Apparat Punkt Nr. 13 in Tab. 1).

Nachdem für einige Punkte $P_{i}$ im $\left(\varphi_{\mathrm{m}}, \varepsilon^{\prime}, \varepsilon^{\prime \prime}\right)$-Raum Lösungen durch Untersuchung der Nullkurven in der $(\vartheta, \delta)$-Ebene gefunden waren, erfolgte die systematische Untersuchung der Umgebung dieser Punkte mit Hilfe des Newtonschen Verfahrens auf weitere Lösungen. Es zeigte sich dabei, daß diese zu Punkten $P_{i}$ gehörigen Lösungen nur für jene Punkte, die den Punkten $P_{i}$ sehr nahe benachbart waren, als Ausgangslösungen verwendet werden konnten. Der erste Schritt von $P_{i}$ aus in $\operatorname{der} \varphi_{\mathrm{m}}$, $\varepsilon^{\prime}$ - oder $\varepsilon^{\prime \prime}$-Richtung wurde stets sehr eng gemacht. Für den nächsten Schritt konnte dann durch lineare Extrapolation eine verbesserte Ausgangslösung ermittelt werden. Beim dritten Schritt in gleicher Richtung erfolgte schließlich quadratische Extrapolation. Nur auf diese Weise konnte eine größere und damit wirtschaftlichere Schrittweite im $\left(\varphi_{\mathrm{m}}, \varepsilon^{\prime}, \varepsilon^{\prime \prime}\right)$-Raum von Lösung zu Lösung erreicht werden.

47 Eckige Klammern kennzeichnen Gleichungen aus der vorangegangenen Arbeit (s. Anm. ${ }^{30}$ ).
Es ist selbstverständlich, daß die Lösungen nicht beliebig genau bestimmt werden konnten. Hinzu kommt, daß aus physikalischen Gründen wegen der in der Rechnung gemachten Voraussetzungen nicht übertrieben genau gerechnet zu werden braucht. Wir bezeichnen daher als Lösung einen solchen Wertesatz $\left(\sqrt{2} \varphi_{\mathrm{e}}, l_{\mathrm{e}}^{\prime} / r_{\mathrm{m}}, l_{\mathrm{m}}^{\prime \prime} / r_{\mathrm{m}}, r_{\mathrm{e}} / r_{\mathrm{m}}, d / r_{\mathrm{m}}\right)$ zu einem vorgegebenen. Wertetripel $\left(\varphi_{\mathrm{m}}, \varepsilon^{\prime}, \varepsilon^{\prime \prime}\right)$, für den

$$
\left|B_{11}\right|+\left|B_{12}\right|<\eta
$$

ist. Aus praktischen Gründen wurde die obere Schranke $\eta=10^{-3}$ gewählt. Sie hätte jedoch im Prinzip beliebig klein vorgegeben werden können.

\section{Kontrolle der Rechnungen}

Eine derart umfangreiche Rechnung erfordert natürlich Kontrollen gegen Fehler. Es wurde daher zu jeder Lösung eine Probe gerechnet. Zur Probe benutzten wir aber die Gln. [20] ${ }^{47}$ der genannten vorangegangenen Arbeit ${ }^{30}$, die unabhängig von den in dieser Arbeit benutzten Ausdrücken der Gln. (8) für $B_{11}, B_{12}$ und $B_{22}$ hergeleitet werden können. Sie lassen sich in die Gln. [15] bzw. Gln. (8) mit Hilfe der Beziehungen [19] umrechnen, wobei die Größen $\mu, v$ und $\mu^{\prime}, v^{\prime}$ der genannten Arbeit nicht bekannt zu sein brauchen. Man überzeugt sich leicht, daß die Gln. [19] dann und nur dann erfüllt sind, wenn die $\mu, \nu$ richtig berechnet sind; denn die $\mu^{\prime}, v^{\prime}$ folgen durch Vertauschen der Indizes aus den $\mu, v$. Aus dem Gelingen der Probe folgt also, daß 1 . die numerische Rechnung richtig durchgeführt wurde, 2. die Gln. [15] und [20] widerspruchsfrei sind und 3 . daß die $\mu, v$ richtig berechnet sind. Die $K$ und $L$ stimmen mit den Ergebnissen von Voorhies ${ }^{9}$ überein. Außerdem lassen sie sich durch Spezialisierung der entsprechenden GröBen des Toroidkondensators herleiten ${ }^{48}$. Damit erscheint uns eine ausreichende Kontrolle sowohl der Rechenergebnisse als auch der Ausgangsformeln gesichert.

\section{Ergebnisse}

\section{Gleichsinnige Ablenkung (Abb. 3)}

Im Falle gleichsinniger Ablenkung wurden zwei Systeme von physikalisch brauchbaren Lösungen gefunden, die nach der willkürlichen Reihenfolge, in der

48 H. Ewald u. H. Liebl, Z. Naturforschg. 10 a, 872 [1955]. 


\begin{tabular}{|c|c|c|c|c|c|c|c|c|c|}
\hline Nr. & $\varphi_{\mathrm{m}}$ & $\varepsilon^{\prime}$ & $\varepsilon^{\prime \prime}$ & $\sqrt{2} \varphi_{\mathrm{e}}$ & $\varphi_{\mathrm{e}}$ & $d / r_{\mathrm{m}}$ & $r_{\mathrm{e}} / r_{\mathrm{m}}$ & $l_{\mathrm{e}}^{\prime} / r_{\mathrm{m}}$ & $l_{\mathrm{m}}^{\prime \prime} / r_{\mathrm{m}}$ \\
\hline \multicolumn{5}{|c|}{ Gleichsinnige Ablenkung } & \multicolumn{5}{|c|}{ 1. Lösungssystem } \\
\hline $\begin{array}{r}1 \\
2 \\
3 \\
4 \\
5 \\
6 \\
7 \\
8 \\
9 \\
10 \\
11 \\
12\end{array}$ & $\begin{array}{r}55 \\
60 \\
60 \\
60 \\
70 \\
72 \\
80 \\
90 \\
105 \\
110 \\
111 \\
116\end{array}$ & $\begin{array}{c}0 \\
0 \\
0 \\
-15 \\
15 \\
15 \\
15 \\
27 \\
32 \\
31 \\
31 \\
35,9\end{array}$ & $\begin{array}{l}0 \\
-3 \\
-15 \\
-15 \\
-15 \\
-15 \\
-15 \\
-15 \\
-35 \\
-35 \\
-35 \\
-45\end{array}$ & $\begin{array}{r}115,272 \\
128,692 \\
80,000 \\
138,380 \\
70,761 \\
83,582 \\
129,381 \\
133,855 \\
65,392 \\
154,540 \\
167,058 \\
182,770\end{array}$ & $\begin{array}{r}81,510 \\
90,999 \\
56,569 \\
97,849 \\
50,036 \\
59,101 \\
91,486 \\
94,650 \\
46,239 \\
109,276 \\
118,128 \\
129,238\end{array}$ & $\begin{array}{l}3,4364 \\
2,9930 \\
4,5200 \\
2,8459 \\
4,9392 \\
4,0507 \\
2,3494 \\
1,5955 \\
3,8878 \\
1,3432 \\
1,2136 \\
1,1190\end{array}$ & $\begin{array}{l}1,2762 \\
1,2203 \\
1,8143 \\
1,0081 \\
2,6663 \\
2,2773 \\
1,5454 \\
1,5938 \\
4,5927 \\
2,0185 \\
1,8983 \\
2,5988\end{array}$ & $\begin{array}{r}0,4768 \\
0,2835 \\
1,8204 \\
0,0879 \\
4,1875 \\
2,5560 \\
0,5406 \\
0,7084 \\
45,8907 \\
0,7614 \\
0,4473 \\
0,7416\end{array}$ & $\begin{array}{l}1,4239 \\
1,2145 \\
0,8377 \\
0,6637 \\
0,9116 \\
0,9082 \\
0,8768 \\
0,9477 \\
0,4426 \\
0,4424 \\
0,4392 \\
0,3174\end{array}$ \\
\hline \multicolumn{5}{|c|}{ Gleichsinnige Ablenkung } & \multicolumn{5}{|c|}{ 2. Lösungssystem } \\
\hline $\begin{array}{l}13 \\
14 \\
15 \\
16 \\
17 \\
18 \\
19 \\
20 \\
21 \\
22\end{array}$ & $\begin{array}{r}60 \\
60 \\
70 \\
72 \\
80 \\
90 \\
90 \\
105 \\
110 \\
115\end{array}$ & $\begin{array}{l}15 \\
23 \\
15 \\
15 \\
33 \\
33 \\
33 \\
32 \\
33 \\
34\end{array}$ & $\begin{array}{l}-15 \\
-15 \\
-15 \\
-15 \\
-15 \\
-15 \\
-30 \\
-35 \\
-45 \\
-40\end{array}$ & $\begin{array}{l}183,220 \\
227,282 \\
140,724 \\
133,451 \\
183,292 \\
128,778 \\
250,548 \\
144,110 \\
158,727 \\
184,544\end{array}$ & $\begin{array}{r}129,556 \\
160,713 \\
99,507 \\
94,364 \\
129,607 \\
91,060 \\
177,164 \\
101,901 \\
112,237 \\
130,492\end{array}$ & $\begin{array}{l}0,9270 \\
0,9449 \\
0,6908 \\
0,6499 \\
0,7162 \\
0,7056 \\
0,7458 \\
1,0577 \\
1,2991 \\
1,0553\end{array}$ & $\begin{array}{l}3,8756 \\
4,5896 \\
3,6209 \\
3,6208 \\
3,3174 \\
2,6338 \\
3,8714 \\
3,1496 \\
3,6810 \\
2,0840\end{array}$ & $\begin{array}{r}5,8805 \\
2,8751 \\
22,1367 \\
45,2327 \\
2,9046 \\
6,3650 \\
0,1440 \\
3,8777 \\
3,1829 \\
0,3143\end{array}$ & $\begin{array}{l}0,9434 \\
0,9870 \\
0,9092 \\
0,9017 \\
0,9528 \\
0,9789 \\
0,5230 \\
0,4463 \\
0,3210 \\
0,3714\end{array}$ \\
\hline
\end{tabular}

Tab. 1. Einige typische der vielen berechneten Lösungen für Apparate mit gleichsinniger Ablenkung, bei denen $B_{11}, B_{12}$ und $B_{22}$ gleichzeitig korrigiert sind (vollständige Doppelfokussierung zweiter Ordnung). Von den angegebenen Apparaten sind die mit den Nummern 1, 8 und 11 in Abb. 10 gezeichnet, die mit den Nummern 18 und 19 in Abb. 11. Die Lösungen 5 und 15,6 und 16 sowie 9 und 20 sind Beispiele für die Mehrdeutigkeit der Lösung zu einem vorgegebenen Wertetripel $\left(\varphi_{\mathrm{m}}, \varepsilon^{\prime}, \varepsilon^{\prime \prime}\right)$. Um Kontrollrechnungen zu ermöglichen, wurden die verschiedenen Werte in der Tabelle mit einer höheren Genauigkeit angegeben als physikalisch notwendig ist.

sie untersucht wurden, mit I und II bezeichnet sind. Innerhalb eines jeden Lösungssystems sind die Komponenten $\sqrt{2} \varphi_{\mathrm{e}}, d / r_{\mathrm{m}}, r_{\mathrm{e}} / r_{\mathrm{m}}, l_{\mathrm{e}}^{\prime} / r_{\mathrm{m}}, l_{\mathrm{m}}^{*} / r_{\mathrm{m}}$ derLösung eine stetige Funktion der Parameter $\varphi_{\mathrm{m}}, \varepsilon^{\prime}, \varepsilon^{\prime \prime}$. Im $\left(\varphi_{\mathrm{m}}, \varepsilon^{\prime}, \varepsilon^{\prime \prime}\right)$-Raum liegen die zu physikalisch sinnvollen Lösungen der Lösungssysteme I und II gehörigen Parameter $\varphi_{\mathrm{m}}, \varepsilon^{\prime}$ und $\varepsilon^{\prime \prime}$ in begrenzten Bereichen $\Pi_{\mathrm{I}}^{+}$und $\Pi_{\mathrm{II}}^{+}$. Über die Größe dieser Bereiche geben die Tab. 1 und auch die Abb. 7, 8 und 9 einen Überblick. Während in Tab. 1 von den vielen ausgerechneten Lösungen nur wenige typische Beispiele angeführt wurden, ist in den Diagrammen 7 , 8 und 9 fast das gesamte Lösungssystem I für gleichsinnige Ablenkung, das für den Bau von Instrumenten hauptsächlich interessiert, enthalten. Durch Interpolation ist es mit Hilfe dieser Diagramme möglich, zumindest näherungsweise auch neue Lösungen zu ermitteln. Wie man der Tab. 1 weiter entnimmt, gibt es Tripel $\left(\varphi_{\mathrm{m}}, \varepsilon^{\prime}, \varepsilon^{\prime \prime}\right)$, zu denen mehr als eine physikalisch sinnvolle Lösung existiert. (Aus der Tatsache, daß zu bestimmten $\varphi_{\mathrm{m}}, \varepsilon^{\prime}, \varepsilon^{\prime \prime}$ zwei Lösungen gefunden wurden, folgt nicht, daß es nur zwei gibt.) Aus dem vorliegenden Material läßt sich schließen, daß die beiden Bereiche $\Pi_{\mathrm{I}}^{+}$und $\Pi_{\mathrm{II}}^{+}$ nicht vollständig zusammenfallen, sich aber teilweise überdecken.

Einige typische Beispiele für den Strahlengang in Instrumenten aus Tab. 1 sind in den Abb. 10 und 11 dargestellt. Es sei noch darauf hingewiesen, daß es in der Umgebung günstiger Lösungen unendlich viele ebenfalls günstige Lösungen gibt.

Von besonderem Interesse ist die Frage des Einflusses geringfügiger Fehler der Apparateabmessungen auf die Korrektur der Bildfehler. Deswegen wurden unter der Voraussetzung, daß Doppelfokussierung erster Ordnung bei einer Dejustierung erhalten bleibt, für einige willkürlich herausgegriffene Beispiele die Bildfehler im Falle ungenauer Justie- 


\begin{tabular}{|c|c|c|c|c|c|c|r|r|r|r|}
\hline$\Delta \varphi_{\mathrm{m}}$ & $\Delta \varepsilon^{\prime}$ & $\Delta \varepsilon^{\prime \prime}$ & $\Delta \varphi_{\mathrm{e}}$ & $\Delta \frac{d}{r_{\mathrm{m}}}$ & $\Delta \frac{r_{\mathrm{e}}}{r_{\mathrm{m}}}$ & $\frac{l_{\mathrm{e}}}{r_{\mathrm{m}}}$ & $\frac{l_{\mathrm{m}}^{\prime \prime}}{r_{\mathrm{m}}}$ & $B_{11}$ & $B_{12}$ & $B_{22}$ \\
\hline 0 & 0 & 0 & 0 & 0 & 0 & 0,284 & 1,214 & 0,000 & 0,000 & 0,000 \\
2 & 0 & 0 & 0 & 0 & 0 & 0,256 & 1,147 & $-0,008$ & 0,384 & $-0,503$ \\
-2 & 0 & 0 & 0 & 0 & 0 & 0,314 & 1,286 & 0,010 & $-0,416$ & 0,503 \\
0 & 2,000 & 0 & 0 & 0 & 0 & 0,267 & 1,294 & 0,082 & $-0,222$ & 0,294 \\
0 & $-2,000$ & 0 & 0 & 0 & 0 & 0,301 & 1,139 & $-0,091$ & 0,251 & $-0,313$ \\
0 & 0 & 2,000 & 0 & 0 & 0 & 0,284 & 1,268 & $-0,013$ & 0,137 & $-0,245$ \\
0 & 0 & $-2,000$ & 0 & 0 & 0 & 0,284 & 1,165 & 0,024 & $-0,184$ & 0,289 \\
0 & 0 & 0 & 2,000 & 0 & 0 & 0,245 & 1,211 & $-0,027$ & 0,064 & $-0,025$ \\
0 & 0 & 0 & $-2,000$ & 0 & 0 & 0,322 & 1,219 & 0,031 & $-0,080$ & 0,036 \\
0 & 0 & 0 & 0 & 0,010 & 0 & 0,280 & 1,213 & $-0,001$ & 0,026 & $-0,023$ \\
0 & 0 & 0 & 0 & 0,060 & 0 & 0,263 & 1,203 & $-0,005$ & 0,156 & $-0,139$ \\
0 & 0 & 0 & 0 & $-0,010$ & 0 & 0,287 & 1,216 & 0,001 & $-0,027$ & 0,023 \\
0 & 0 & 0 & 0 & $-0,060$ & 0 & 0,304 & 1,226 & 0,005 & $-0,161$ & 0,135 \\
0 & 0 & 0 & 0 & 0 & 0,010 & 0,296 & 1,212 & $-0,026$ & 0,028 & $-0,031$ \\
0 & 0 & 0 & 0 & 0 & 0,060 & 0,361 & 1,198 & $-0,158$ & 0,182 & $-0,190$ \\
0 & 0 & 0 & 0 & 0 & 0,010 & 0,271 & 1,217 & 0,024 & $-0,028$ \\
0 & 0 & 0 & 0 & 0 & 0,060 & 0,213 & 1,232 & 0,143 & $-0,152$ & 0,031 \\
\hline
\end{tabular}

Tab. 2. Übersicht über die Werte von $l_{\mathrm{e}}{ }^{\prime} / r_{\mathrm{m}}, l_{\mathrm{m}}{ }^{\prime \prime} / r_{\mathrm{m}}, B_{11}, B_{12}, B_{22}$, die sich ergeben, wenn man bei einem von zweiter Ordnung doppelfokussierenden Apparat $\varphi_{\mathrm{m}}, \varepsilon^{\prime}, \varepsilon^{\prime \prime}, \sqrt{2} \varphi_{\mathrm{e}}, d / r_{\mathrm{m}}, r_{\mathrm{e}} / r_{\mathrm{m}}$ willkürlich um die angegebenen Beträge $\Delta x\left(x=\varphi_{\mathrm{m}}, \varepsilon^{\prime}\right.$, $\left.\varepsilon^{\prime \prime}, \varphi_{\mathrm{e}}, d / r_{\mathrm{m}}, r_{\mathrm{e}} / r_{\mathrm{m}}\right)$ ändert, wobei für den falsch justierten Apparat Doppelfokussierung erster Ordnung gefordert wird. Im vorliegenden Beispiel ist $\varphi_{\mathrm{m}}=60^{\circ}, \varepsilon^{\prime}=0, \varepsilon^{\prime \prime}=-3^{\circ}, \sqrt{2} \varphi_{\mathrm{e}}=128,692, d / r_{\mathrm{m}}=2,993, r_{\mathrm{e}} / r_{\mathrm{m}}=1,220$. Wie man sieht, bleiben die Bildfehler noch klein, wenn die Winkel um $1^{\circ}$, die Längen um $0,01 r_{\mathrm{m}}$ falsch gewählt werden.

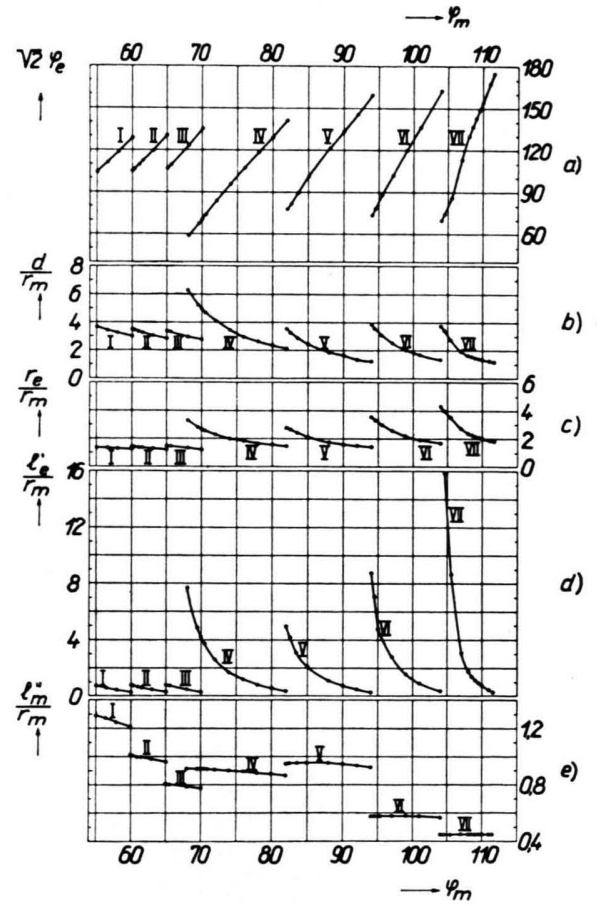

Abb. 7 a-e. Die Abhängigkeit von $\varphi_{\mathrm{m}}$ der Komponenten $\sqrt{2} \varphi_{\mathrm{e}}, d / r_{\mathrm{m}}, r_{\mathrm{e}} / r_{\mathrm{m}}, l_{\mathrm{e}}^{\prime} / r_{\mathrm{m}}$ und $l_{\mathrm{m}}{ }^{\prime \prime} / r_{\mathrm{m}}$ der Lösung bei konstantem $\varepsilon^{\prime}$ und $\varepsilon^{\prime \prime}$. Die Numerierung der Kurven bedeutet

\begin{tabular}{|c|c|c|c|c|c|c|c|}
\hline & I & II & III & IV & V & VI & VII \\
\hline$\varepsilon^{\prime}$ & $0^{\circ}$ & $0^{\circ}$ & $0^{\circ}$ & $15^{\circ}$ & $27^{\circ}$ & $27^{\circ}$ & $31^{\circ}$ \\
$\varepsilon^{\prime \prime}$ & $-3^{\circ}$ & $-9^{\circ}$ & $-15^{\circ}$ & $-15^{\circ}$ & $-15^{\circ}$ & $-27,5^{\circ}$ & $-35^{\circ}$ \\
\hline
\end{tabular}

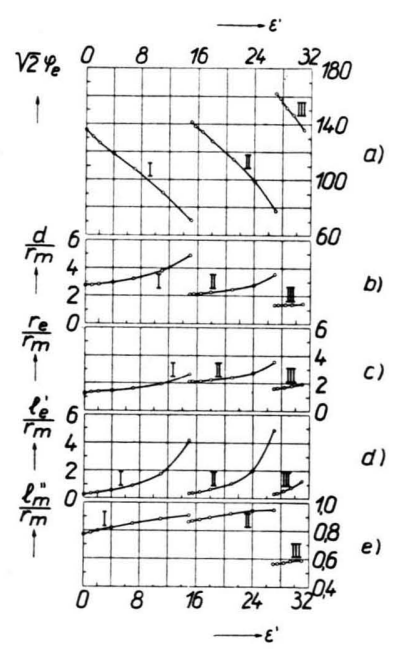

Abb. $8 \mathrm{a}-$ e. Die Abhängigkeit von $\varepsilon^{\prime}$ der Komponenten $\sqrt{2} \varphi_{\mathrm{e}}, d / r_{\mathrm{m}}, r_{\mathrm{e}} / r_{\mathrm{m}}, l_{\mathrm{e}}^{\prime} / r_{\mathrm{m}}, l_{\mathrm{m}}^{\prime \prime} / r_{\mathrm{m}}$ der Lösung bei konstantem $\varphi_{\mathrm{m}}$ und $\varepsilon^{\prime \prime}$. Die Numerierung der Kurven bedeutet

\begin{tabular}{|c|c|c|c|}
\hline & I & II & III \\
\hline$\varphi_{\mathrm{m}}$ & $70^{\circ}$ & $82^{\circ}$ & $104^{\circ}$ \\
$\varepsilon^{\prime \prime}$ & $-15^{\circ}$ & $-15^{\circ}$ & $-27,5^{\circ}$ \\
\hline
\end{tabular}

rung berechnet. In der Tab. 2 sind die Ergebnisse für ein Beispiel zusammengefaßt. Wie man sieht, bleiben die Bildfehler noch klein, wenn die Winkel um $2^{\circ}$, die Längen um Prozente falsch gewählt werden. 


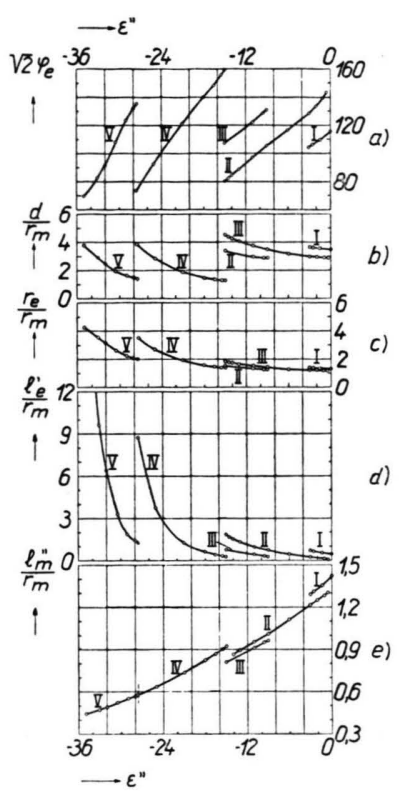

Abb. 9 a-e. Die Abhängigkeit von $\varepsilon^{\prime \prime}$ der Komponenten $\sqrt{ } 2 \varphi_{\mathrm{e}}, d / r_{\mathrm{m}}, r_{\mathrm{e}} / r_{\mathrm{m}}, l_{\mathrm{e}}^{\prime} / r_{\mathrm{m}}, l_{\mathrm{m}}^{\prime \prime} / r_{\mathrm{m}}$ der Lösung bei konstantem $\varphi_{\mathrm{m}}$ und $\varepsilon^{\prime}$. Die Numerierung der Kurven bedeutet

\begin{tabular}{|cccccc|}
\hline & I & II & III & IV & V \\
$\varphi_{\mathrm{m}}$ & $55^{\circ}$ & $60^{\circ}$ & $65^{\circ}$ & $94^{\circ}$ & $104^{\circ}$ \\
$\varepsilon^{\prime}$ & $0^{\circ}$ & $0^{\circ}$ & $0^{\circ}$ & $27^{\circ}$ & $31^{\circ}$ \\
\hline
\end{tabular}

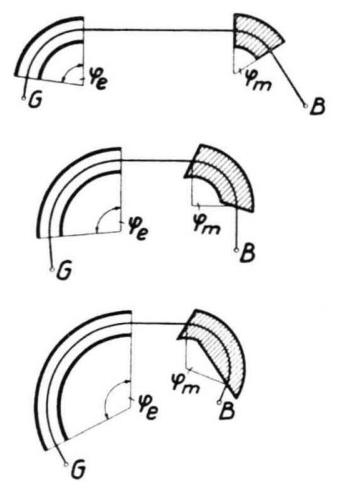

Abb. 10. Drei Apparate mit Doppelfokussierung zweiter Ordnung aus dem ersten Lösungssystem im Fall gleichsinniger Ablenkung. Die Ablenkwinkel im Magnetfeld sind $\varphi_{\mathrm{m}}=55^{\circ}$, $90^{\circ}$ und $111^{\circ}$. Die übrigen Daten sind aus Tab. 1 und der zugehörigen Unterschrift zu entnehmen.

\section{Gegensinnige Ablenkung (Abb.4)}

Ebenso wie im Falle gleichsinniger Ablenkung gibt es im Falle gegensinniger Ablenkung physikalisch sinnvolle Lösungen. Wiederum wurden zwei
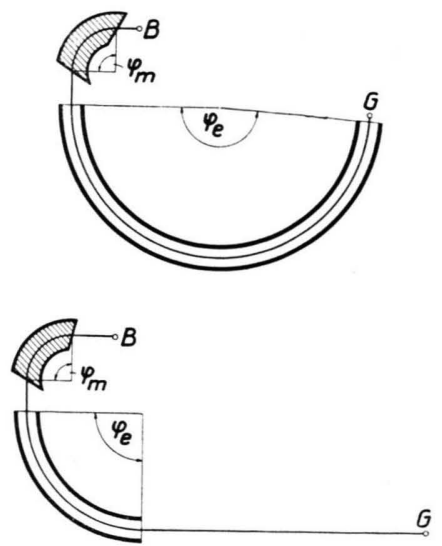

Abb. 11. Zwei Apparate mit Doppelfokussierung zweiter Ordnung aus dem zweiten Lösungssystem im Fall gleichsinniger Ablenkung. Die Ablenkwinkel im Magnetfeld betragen $90^{\circ}$.

Die übrigen Daten sind aus Tab. 1 und der zugehörigen Unterschrift zu entnehmen.

Lösungsbereiche $\Pi_{\mathrm{I}}^{-}$und $\Pi_{\mathrm{II}}^{-}$gefunden, innerhalb welcher die Lösungen stetig von den Parametern $\varphi_{\mathrm{m}}, \varepsilon^{\prime}$ und $\varepsilon^{\prime \prime}$ abhängen. Es sind auch hier Fälle vorhanden, für die zu einem Wertetripel $\left(\varphi_{\mathrm{m}}, \varepsilon^{\prime}, \varepsilon^{\prime \prime}\right)$ mehr als eine physikalisch sinnvolle Lösung existiert.

Die Ergebnisse sind in Tab. 3 zusammengefaßt. Wie man sieht, haben die Massenspektrometer mit korrigierten Bildfehlern bei gegensinniger Ablenkung nicht in gleicher Weise angenehme Abmessungen wie im Fall gleichsinniger Ablenkung. In den Abb. 12 und 13 sind zwei Beispiele dargestellt.

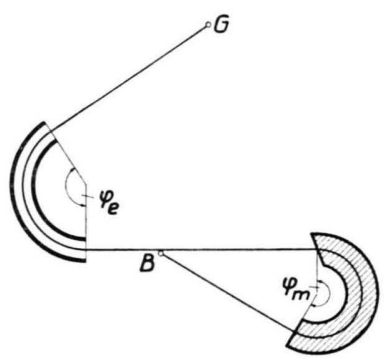

Abb. 12. Ein von zweiter Ordnung doppelfokussierender Apparat mit dem Ablenkwinkel im Magnetfeld $\varphi_{\mathrm{m}}=210^{\circ}$ aus dem ersten Lösungssystem im Fall gegensinniger Ablenkung. Die übrigen Daten enthält Tab. 3.

\section{Die vollständigen Rechenergebnisse}

In der vorliegenden Arbeit konnte wegen Platzmangel nur ein kleiner Bruchteil der gewonnenen Rechenergebnisse wiedergegeben werden. So wurden z. B. etwa 380 Apparate mit Doppelfokussierung zweiter Ordnung berechnet. Erst recht mußten die 


\begin{tabular}{|c|c|c|c|c|c|c|c|c|c|}
\hline Nr. & $\varphi_{\mathrm{m}}$ & $\varepsilon^{\prime}$ & $\varepsilon^{\prime \prime}$ & $\sqrt{2} \varphi_{\mathrm{e}}$ & $\varphi_{\mathrm{e}}$ & $d / r_{\mathrm{m}}$ & $r_{\mathrm{e}} / r_{\mathrm{m}}$ & $l_{\mathrm{e}}^{\prime} / r_{\mathrm{m}}$ & $l_{\mathrm{m}}^{\prime \prime} / r_{\mathrm{m}}$ \\
\hline \multicolumn{5}{|c|}{ Gegensinnige Ablenkung } & \multicolumn{5}{|c|}{ 1. Lösungssystem } \\
\hline $\begin{array}{l}1 \\
2 \\
3 \\
4 \\
5 \\
6 \\
7 \\
8\end{array}$ & $\begin{array}{l}196 \\
197 \\
200 \\
210 \\
210 \\
210 \\
210 \\
211\end{array}$ & $\begin{array}{l}-19,5 \\
-19,5 \\
-19,5 \\
-20 \\
-26 \\
-32 \\
-32 \\
-19,5\end{array}$ & $\begin{array}{l}0 \\
0 \\
0 \\
0 \\
0 \\
0 \\
8 \\
0\end{array}$ & $\begin{array}{l}196,853 \\
197,690 \\
200,108 \\
207,349 \\
207,958 \\
208,252 \\
195,167 \\
207,929\end{array}$ & $\begin{array}{l}139,196 \\
139,788 \\
141,498 \\
146,618 \\
147,049 \\
147,256 \\
138,004 \\
147,028\end{array}$ & $\begin{array}{l}6,6206 \\
6,5723 \\
6,4211 \\
5,7939 \\
5,0098 \\
4,4167 \\
3,5530 \\
5,8144\end{array}$ & $\begin{array}{l}0,9686 \\
1,0147 \\
1,1434 \\
1,4569 \\
1,3098 \\
1,1894 \\
4,6347 \\
1,4954\end{array}$ & $\begin{array}{l}4,6671 \\
4,6555 \\
4,6071 \\
4,2861 \\
3,7867 \\
3,4205 \\
2,4993 \\
4,3029\end{array}$ & $\begin{array}{l}6,9003 \\
6,4834 \\
5,4829 \\
3,5846 \\
3,5099 \\
3,4278 \\
7,4693 \\
3,4681\end{array}$ \\
\hline \multicolumn{6}{|c|}{ Gegensinnige Ablenkung } & \multicolumn{3}{|c|}{ 2. Lösungssystem } & \\
\hline $\begin{array}{r}9 \\
10 \\
11 \\
12 \\
13 \\
14 \\
15 \\
16\end{array}$ & $\begin{array}{l}177 \\
180 \\
180 \\
180 \\
180 \\
189 \\
195 \\
197\end{array}$ & $\begin{array}{l}-19,5 \\
-19 \\
-34 \\
-35 \\
-35 \\
-19,5 \\
-19,5 \\
-19,5\end{array}$ & $\begin{array}{c}0 \\
0 \\
0 \\
0,5 \\
-12,5 \\
0 \\
0 \\
0\end{array}$ & $\begin{array}{l}213,447 \\
205,263 \\
155,254 \\
151,429 \\
239,683 \\
178,108 \\
165,716 \\
162,302\end{array}$ & $\begin{array}{l}150,930 \\
145,143 \\
109,781 \\
107,076 \\
169,481 \\
125,941 \\
117,179 \\
114,765\end{array}$ & $\begin{array}{l}6,8342 \\
5,6462 \\
1,7517 \\
1,6115 \\
2,4853 \\
3,1831 \\
2,4910 \\
2,3215\end{array}$ & $\begin{array}{l}8,6135 \\
6,8927 \\
2,8409 \\
2,6709 \\
4,1779 \\
3,7284 \\
2,8218 \\
2,6020\end{array}$ & $\begin{array}{r}10,4646 \\
9,4120 \\
8,7832 \\
9,7907 \\
1,3164 \\
8,2417 \\
8,9651 \\
9,4402\end{array}$ & $\begin{array}{l}3,2699 \\
2,9668 \\
2,2471 \\
2,2973 \\
1,1265 \\
2,2943 \\
2,0287 \\
1,9547\end{array}$ \\
\hline
\end{tabular}

Tab 3. Einige typische der vielen berechneten Lösungen für Apparate mit gegensinniger Ablenkung, bei denen $B_{11}, B_{12}$, und $B_{22}$ gleichzeitig korrigiert sind (vollständige Doppelfokussierung zweiter Ordnung). Von den angegebenen Apparaten ist der mit der Nummer 4 in Abb. 12, der mit der Nummer 13 in Abb. 13 gezeichnet. Apparat 2 und Apparat 16 stellen ein Beispiel für die Mehrdeutigkeit der Lösung zu einem vorgegebenen Wertetripel $\left(\varphi_{\mathrm{m}}, \varepsilon^{\prime}, \varepsilon^{\prime \prime}\right)$ dar.

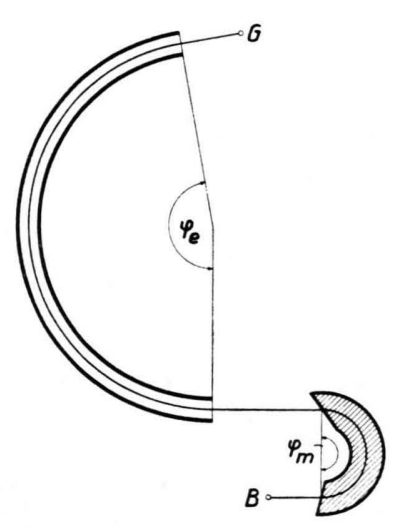

Abb. 13. Ein von zweiter Ordnung doppelfokussierender Apparat mit dem Ablenkwinkel im Magnetfeld $\varphi_{\mathrm{m}}=180^{\circ}$ aus dem zweiten Lösungssystem im Fall gegensinniger Ablenkung. Die übrigen Daten enthält Tab. 3 .

Ergebnisse der vorbereitenden Rechnungen weggelassen werden. Hiervon ist besonders die Tabellierung der Koeffizienten $\varkappa$ und $\lambda$ für die Bahnen nach erfolgter Ablenkung im elektrischen. Radialfeld $\left(30^{\circ} \leqq \sqrt{ } 2 \varphi_{\mathrm{e}} \leqq 180^{\circ} ;\right.$ Schrittweite $\left.1^{\circ}\right)$ sowie der Koeffizienten $u$ und $v$ für die Bahnen nach erfolgter Ablenkung im Magnetfeld $\left(30^{\circ} \leqq \varphi_{\mathrm{m}} \leqq 210^{\circ}\right.$; $-40^{\circ} \leqq \varepsilon^{\prime} \leqq 40^{\circ} ; \quad-40^{\circ} \leqq \varepsilon^{\prime \prime} \leqq 40^{\circ} ; \quad$ Schrittweite für $\varphi_{\mathrm{m}}, \varepsilon^{\prime}$ und $\varepsilon^{\prime \prime} 10^{\circ}$ ) zu erwähnen. Die genannten Ergebnisse liegen in Form von Lochkarten bei uns vor und können für Interessenten gegen Erstattung der Unkosten für die Vervielfältigung auf Wunsch mit einer IBM-Tabelliermaschine geschrieben werden.

Zum Schluß möchten wir Herrn Professor Mattauch für die Bereitstellung außertourlicher Institutsmittel zur Durchführung der Rechnung herzlich danken. Ferner danken wir den Herren K. Brokate und Dr. K. Hesse vom Rechenzentrum der IBM Deutschland, Sindelfingen, für ihr besonderes Entgegenkommen bei der Durchführung der Rechnungen und insbesondere Fräulein Dr. G. Beyer für die Durchführung der umfangreichen Programmierungsarbeiten sowie für ihre Mitarbeit bei der Auswertung der während der numerischen Rechnung anfallenden Zwischenergebnisse. Auch Fräulein BraUM und den Herren Heinrichs, Mehnert und Stichel möchten wir für die Durchführung umfangreicher Kontrollrechnungen mit der Tischrechenmaschine danken. 